\title{
DEFORMATION OF SASAKIAN METRICS
}

\author{
HIRAKU NOZAWA
}

\begin{abstract}
Deformations of the Reeb flow of a Sasakian manifold as transversely Kähler flows may not admit compatible Sasakian metrics. We show that the triviality of the $(0,2)$-component of the basic Euler class characterizes the existence of compatible Sasakian metrics for given small deformations of the Reeb flow as transversely holomorphic Riemannian flows. We also prove a Kodaira-Akizuki-Nakano type vanishing theorem for basic Dolbeault cohomology of homologically orientable transversely Kähler foliations. As a consequence of these results, we show that any small deformations of the Reeb flow of a positive Sasakian manifold admit compatible Sasakian metrics.
\end{abstract}

\section{Contents}

1. Introduction

2. Sasakian structures and their underlying structures

3. Basic Euler classes of transversely holomorphic isometric flows

4. Existence of extensions of Sasakian metrics

5. Stability of K-contact structures for transversely Kähler foliations with a fixed underlying transverse Kähler flow

\section{INTRODUCTION}

1.1. Characterization of the stability of Sasakian metrics. The Reeb flow of the contact form $\eta$ of a Sasakian manifold has a transverse Kähler structure, which is one of the important underlying structures of Sasakian manifolds as well as $C R$ structures. In this paper, we investigate under which conditions given families of deformation of the Reeb flow of $\eta$ as transversely holomorphic Riemannian flows have compatible Sasakian metrics. Although any small deformations of the complex

Received by the editors September 15, 2010 and, in revised form, October 6, 2011 and October 3,2012 .

2010 Mathematics Subject Classification. Primary 32G07; Secondary 53C25.

Key words and phrases. Deformation theory, Sasakian metrics, transversely holomorphic foliations.

The author was partially supported by Grant-in-Aid for JSPS Fellows (19-4609) and Postdoctoral Fellowship of French government (662014L). 
structure of a compact Kähler manifold admit compatible Kähler metrics by a theorem of Kodaira and Spencer [33, there exists a Sasakian manifold whose Reeb flow can be deformed so that it does not admit a compatible Sasakian metric. We present an example of such a Sasakian metric on a circle bundle over a complex torus in Section 8.2. Seeing the example we note that the nontriviality of the $(0,2)$ component of the basic Euler class of flows gives an obstruction for the existence of compatible Sasakian metrics. Our main result shows that this obstruction is unique, that is, small deformations of the Reeb flow admit compatible Sasakian metrics, if the basic Euler class is of degree $(1,1)$. Note that Boyer and Galicki discussed a similar problem in [8, Section 8.2]. Van Coevering [13] proved the stability of Sasaki-extremal and Sasaki-Einstein metrics by using our main result in a part of his proof. The product of $S^{1}$ with a Sasakian manifold admits geometric structures called Vaisman manifolds or locally conformal Kähler manifolds (see Ornea and Verbitsky [39]). Belgun [5] showed that both of these structures are not stable under small deformations of complex structures. Note that Ornea and Verbitsky [40] discovered the stability of another class of geometric structure under small deformations of complex structures, which they call locally conformal Kähler manifolds with potential.

A 1-dimensional foliation is called a flow in this article in accordance with the references. Our main result is stated as follows: Let $V$ be an open neighborhood of 0 in $\mathbb{R}^{\ell}$ and $\left\{\left(\mathcal{F}^{t}, J^{t}, g_{\nu}^{t}\right)\right\}_{t \in V}$ be a smooth family of transversely holomorphic Riemannian flows on a closed manifold $M$. Assume that $\left(\mathcal{F}^{0}, J^{0}, g_{\nu}^{0}\right)$ is the underlying transversely Kähler structure of the Reeb flow of a Sasakian metric $(g, \eta)$.

Theorem 1.1. If the basic Euler class of $\left(\mathcal{F}^{t}, J^{t}\right)$ is of degree $(1,1)$ for every $t$ in $V$, then there exists an open neighborhood $V_{1}$ of 0 in $V$ and a smooth family of Sasakian metrics $\left\{\left(g^{t}, \eta^{t}\right)\right\}_{t \in V_{1}}$ on $M$ such that the underlying transversely holomorphic flow of the Reeb flow of $\left(g^{t}, \eta^{t}\right)$ is $\left(\mathcal{F}^{t}, J^{t}\right)$ for every $t$ in $V_{1}$ and $\left(g^{0}, \eta^{0}\right)=(g, \eta)$.

For the existence of a smooth family of compatible Sasakian metrics, it is necessary that the basic Euler class of $\left(\mathcal{F}^{t}, J^{t}\right)$ is of degree $(1,1)$ (see Lemma 3.19). In this sense, Theorem 1.1 characterizes the stability of the Sasakian metric $(g, \eta)$.

The difficulty in proving Theorem 1.1 comes from the noncontinuous change of the complex of basic differential forms of foliations. The families of the partial differential equation that we need to solve to prove Theorem 1.1 is discontinuous with respect to the parameter $t$ because of this discontinuous change of the basic de Rham complex. To avoid this difficulty, we will apply the invariance of geometric tautness of Riemannian flows obtained in [38]. This result allows us to convert problems on families of basic de Rham complexes to problems on families of the de Rham complexes, where we can apply the Kodaira-Spencer theory [33] on smooth families of self-adjoint strongly elliptic differential operators.

1.2. Vanishing theorem for basic cohomology of positive Sasakian manifolds. We show the following result, which was independently obtained by Goto 24]:

Theorem 1.2. Let $(M, \mathcal{F})$ be the underlying transversely Kähler flow of a positive Sasakian manifold. Then we have

$$
H_{b}^{0, q}(M / \mathcal{F})=0
$$


for $q>0$ and

$$
H_{b}^{p, 0}(M / \mathcal{F})=0
$$

for $p>0$.

Theorem 1.2 is a generalization of a part of Proposition 2.4 of Boyer, Galicki and Nakamaye 10, where they assume that Sasakian manifolds are quasi-regular, that is, every leaf of $\mathcal{F}$ is closed. In this case, the leaf space $M / \mathcal{F}$ is a complex orbifold. Thus they deduced Theorem 1.2 from the Kodaira-Baily vanishing theorem for complex orbifolds due to Baily [4].

To show Theorem 1.2 for general positive Sasakian manifolds, there are two problems: The first problem is that the Kodaira-Akizuki-Nakano vanishing theorem was not known for general Sasakian manifolds whose Reeb flow has a nonclosed orbit. The second problem is that $H_{b}^{p, q}(M / \mathcal{F}, E)$ may not be isomorphic to the sheaf cohomology $H^{q}\left(M, \Omega_{b}^{p} \otimes E\right)$, where $\Omega_{b}^{p}$ is the sheaf of basic holomorphic $p$ forms on $(M, \mathcal{F})$ as we will mention in Remark 3.11. Because of these difficulties, the classical argument to show Theorem 1.2 for Fano manifolds did not work for basic cohomology of positive Sasakian manifolds. The second problem was pointed out by Boyer, Galicki and Nakamaye before Proposition 2.4 in [10]. We show Theorem 1.2 by solving these two problems: We will show that the Kodaira-Akizuki-Nakano vanishing theorem is true for general homologically orientable transversely Kähler foliations as mentioned in the next section. We will solve the second problem by a simple observation on isomorphisms between the spaces of harmonic forms (see Section 6.11).

\subsection{Kodaira-Akizuki-Nakano vanishing theorem for transversely Kähler}

foliations. Let $(M, \mathcal{F})$ be a closed manifold with a homologically orientable transversely Kähler foliation of complex codimension $n$. Let $E$ be an $\mathcal{F}$-fibered Hermitian holomorphic line bundle over $(M, \mathcal{F})$.

Theorem 1.3. If $E$ is positive, then

$$
H_{b}^{p, q}(M / \mathcal{F}, E)=0
$$

for $p+q>n$.

In the special case where the leaves of $\mathcal{F}$ are compact, Theorem 1.3 is the Kodaira-Baily vanishing theorem [4. Theorem 1.3] is proved by the application of the work of El Kacimi Alaoui and Hector [20] and El Kacimi Alaoui [19] on transversely elliptic operators on Riemannian foliations. Their theory allows us to reduce the proof to consider differential operators on transversals of foliations, where the classical argument of Nakano-Akizuki [1] for Kähler manifolds can be applied following Demailly [14.

1.4. Stability of positive Sasakian metrics. We obtain the following corollary of Theorems 1.1 and 1.2 Let $V$ be an open neighborhood of 0 in $\mathbb{R}^{\ell}$ and $\left\{\left(\mathcal{F}^{t}, J^{t}\right)\right\}_{t \in V}$ be a smooth family of transversely holomorphic Riemannian flows on a closed manifold $M$. Assume that $\left(\mathcal{F}^{0}, J^{0}, g_{\nu}^{0}\right)$ is the underlying transversely holomorphic structure of the Reeb flow of a positive Sasakian metric $(g, \eta)$.

Corollary 1.4. There exists an open neighborhood $V_{1}$ of 0 in $V$ and a smooth family of Sasakian metrics $\left\{\left(g^{t}, \eta^{t}\right)\right\}_{t \in V_{1}}$ on $M$ such that $g^{t}$ is a Sasakian metric such that the underlying transversely holomorphic flow of the Reeb flow of $\left(g^{t}, \eta^{t}\right)$ is $\left(\mathcal{F}^{t}, J^{t}\right)$ for every $t$ in $V_{1}$ and $\left(g^{0}, \eta^{0}\right)=(g, \eta)$. 
Corollary 1.4 is deduced from Theorems 1.1 and 1.2 as follows: Since the positivity of the anticanonical line bundles of transversely holomorphic foliations is preserved under small deformation, the assumption of the positivity of $(g, \eta)$ implies that $H_{b}^{0,2}\left(M / \mathcal{F}^{t}\right)=0$ for $t$ in an open neighborhood $V^{\prime}$ of 0 in $V$ by Theorem 1.2 . Hence the basic Euler class of $\left(\mathcal{F}^{t}, J^{t}\right)$ is of degree $(1,1)$ for $t$ in $V^{\prime}$ by definition. Thus Theorem 1.1 concludes the proof.

1.5. Stability of $K$-contact structures in families of Riemannian flows. We obtain a $K$-contact variant of Theorem 1.1 Let $M$ be a closed manifold. Let $(g, \eta)$ be a $K$-contact structure on $M$ (see Definition 2.12 for $K$-contact structures). Let $V$ be an open neighborhood of 0 in $\mathbb{R}^{\ell}$. Let $\left\{\mathcal{F}^{t}\right\}_{t \in V}$ be a smooth family of Riemannian flows on $M$ such that $\mathcal{F}^{0}$ is the Reeb flow of $\eta$.

Theorem 1.5. There exists an open neighborhood $V_{1}$ of 0 in $V$ and a smooth family $\left\{\left(g^{t}, \eta^{t}\right)\right\}_{t \in V_{1}}$ of $K$-contact structures on $M$ such that $\mathcal{F}^{t}$ is the Reeb flow of $\eta^{t}$ for every $t$ in $V_{1}$ and $\eta^{0}=\eta$.

1.6. Moduli space of Sasakian metrics with a fixed transversely Kähler flow. We describes the difference between moduli spaces of Sasakian metrics and their underlying transversely Kähler flows. Let $M$ be a closed manifold. Let $\mathcal{S}$ be the set of Sasakian metrics on $M$. We assume that $\mathcal{S}$ is nonempty. Let $\mathcal{K}$ be the isomorphism classes of transversely Kähler flows on $M$. There exists a natural $\operatorname{map} \mathcal{M}: \mathcal{S} \rightarrow \mathcal{K}$, which maps each Sasakian metric to the isomorphism class of the underlying transversely Kähler flow. We take a point $\mathfrak{k}_{0}$ on $\mathcal{K}$. Let $\operatorname{Diff}\left(\mathfrak{k}_{0}\right)$ be the subgroup of $\operatorname{Diff}(M)$ consisting of diffeomorphisms which preserve the transversely Kähler flow $\mathfrak{k}_{0}$. Let $\operatorname{Diff}_{0}\left(\mathfrak{k}_{0}\right)$ be the identity component of Diff $\left(\mathfrak{k}_{0}\right)$ with the Fréchet topology. Note that $\eta-f^{*} \eta$ is a closed form for $f$ in $\operatorname{Diff}_{0}\left(\mathfrak{k}_{0}\right)$ because $f$ preserves the transverse Kähler form $d \eta$. We define

$$
\operatorname{Ham}\left(\mathfrak{k}_{0}\right)=\left\{f \in \operatorname{Diff}_{0}\left(\mathfrak{k}_{0}\right) \mid\left[\eta-f^{*} \eta\right]=0 \in H^{1}(M ; \mathbb{R})\right\} .
$$

Then we have

Theorem 1.6. There exists a homeomorphism

$$
\mathcal{M}^{-1}\left(\mathfrak{k}_{0}\right) / \operatorname{Ham}\left(\mathfrak{k}_{0}\right) \longrightarrow H^{1}(M ; \mathbb{R}) .
$$

Thus we have the following

Corollary 1.7. Let $M$ be a closed manifold whose first Betti number is zero. If the underlying transversely Kähler flows of two Sasakian metrics $\left(g_{1}, \alpha_{1}\right)$ and $\left(g_{2}, \alpha_{2}\right)$ are isomorphic, then $\left(M, g_{1}, \alpha_{1}\right)$ and $\left(M, g_{2}, \alpha_{2}\right)$ are isomorphic.

Note that $\operatorname{Diff}_{0}\left(\mathfrak{k}_{0}\right) / \operatorname{Ham}\left(\mathfrak{k}_{0}\right)$ is an abelian group (see the second paragraph of Section (7). Thus $\mathcal{M}^{-1}\left(\mathfrak{k}_{0}\right) / \operatorname{Diff}_{0}\left(\mathfrak{k}_{0}\right)$ is a quotient of a vector space by an abelian group in general.

\section{SAsakian STRUCTURES AND THEIR UNDERLYing STRUCTURES}

2.1. Sasakian metrics. Let $M$ be an odd-dimensional smooth manifold. We recall

Definition 2.1. A pair of a contact form $\eta$ and a Riemannian metric $g$ on $M$ is a Sasakian metric on $M$ if the Riemannian metric $r^{2} g+d r \otimes d r$ on $M \times \mathbb{R}_{>0}$ is a Kähler metric with Kähler form $d\left(r^{2} \eta\right)$, where $r$ is the standard coordinate on $\mathbb{R}_{>0}$. 
Basic examples of Sasakian manifolds are circle bundles associated to positive holomorphic line bundles over Kähler manifolds, links of isolated singularities of complex hypersurfaces defined by weighted homogeneous polynomials and contact toric manifolds of Reeb type (see Boyer and Galicki [7] and Blair [6]).

2.2. Foliations with transverse structures. The Reeb flow $\mathcal{F}$ of a Sasakian manifold $(M, \eta, g)$ is the orbit foliation of the flow on $M$ generated by the Reeb vector field $\xi$ of $\eta$, which is defined by equations $\eta(\xi)=1$ and $\iota_{\xi} d \eta=0$. In Section 2.2, we recall the definition of certain transverse structures of $\mathcal{F}$ which are given by the Sasakian metric.

We denote the tangent bundle of $\mathcal{F}$ by $T \mathcal{F}$. By the integrability of $\mathcal{F}$, the Lie bracket on $C^{\infty}(T M)$ induces the Lie derivative with respect to vector fields tangent to the leaves

$$
\begin{aligned}
C^{\infty}(T \mathcal{F}) \otimes C^{\infty}\left((T M / T \mathcal{F})^{\otimes r} \otimes\left(T^{*} M / T^{*} \mathcal{F}\right)^{\otimes s}\right) \\
\longrightarrow C^{\infty}\left((T M / T \mathcal{F})^{\otimes r} \otimes\left(T^{*} M / T^{*} \mathcal{F}\right)^{\otimes s}\right)
\end{aligned}
$$

for every $r \geq 0$ and $s \geq 0$.

Definition 2.2. A foliation $\mathcal{F}$ of $M$ with a tensor $g_{\nu}$ in $C^{\infty}\left(\left(T^{*} M / T^{*} \mathcal{F}\right)^{\otimes 2}\right)$ is called a Riemannian foliation of $M$ if

(i) $g_{\nu}$ is symmetric, positive definite and

(ii) $\mathcal{L}_{Z} g_{\nu}=0$ for every $Z$ in $C^{\infty}(T \mathcal{F})$.

We call such $g_{\nu}$ a transverse metric of $\mathcal{F}$. A Riemannian foliation of dimension one is called a Riemannian flow.

Example 2.3. A foliation defined by a one parameter family of isometries of a Riemannian manifold is a Riemannian flow.

We recall some terminology to define transversely holomorphic foliations.

Definition 2.4. A local vector field $X$ of $T M$ is basic with respect to $\mathcal{F}$ if $[Y, X]$ is tangent to $\mathcal{F}$ for every local vector field $Y$ tangent to $\mathcal{F}$.

It is easy to see that a local vector field $X$ is basic if and only if the flow generated by $X$ maps each leaf of $\mathcal{F}$ to a leaf of $\mathcal{F}$. Let $\pi: T M \rightarrow T M / T \mathcal{F}$ be the canonical projection.

Definition 2.5. A local section $X$ of $T M / T \mathcal{F}$ is transverse with respect to $\mathcal{F}$ if $\pi(\tilde{X})=X$ for some basic local vector field with respect to $\mathcal{F}$.

The subspace $\mathcal{X}(\mathcal{F})$ of $C^{\infty}(T M)$ consisting of basic vector fields is a Lie subalgebra of $C^{\infty}(T M)$. The subspace of $C^{\infty}(T M / T \mathcal{F})$ consisting of transverse vector fields admits a Lie bracket $[\cdot, \cdot]$ which satisfies $[\pi(\tilde{X}), \pi(\tilde{Y})]=\pi[\tilde{X}, \tilde{Y}]$ for any basic vector fields $\widetilde{X}$ and $\tilde{Y}$, because $C^{\infty}(T \mathcal{F})$ is an ideal of $\mathcal{X}(\mathcal{F})$. We recall

Definition 2.6. A foliation $\mathcal{F}$ of $M$ with a tensor $J$ in $\operatorname{Aut}(T M / T \mathcal{F})$ is called a transversely holomorphic foliation of $M$ if

(i) $J^{2}=-$ id,

(ii) $\mathcal{L}_{Z} J=0$ for every $Z$ in $C^{\infty}(T \mathcal{F})$ and

(iii) the Nijenhaus tensor $N_{J}(X, Y)=[J X, J Y]-J[J X, Y]-J[X, J Y]-[X, Y]$ of $J$ vanishes on any local transverse vector fields $X$ and $Y$ with respect to $\mathcal{F}$. 
We call such $J$ a transverse complex structure of $\mathcal{F}$. A transversely holomorphic foliation of dimension one is called a transversely holomorphic flow.

Example 2.7. Let $B^{n}$ be the unit disk of $\mathbb{C}^{n}$. Consider a foliation $\mathcal{F}_{\text {std }}$ on $\mathbb{R}^{m} \times B^{n}$ given by a decomposition $\mathbb{R}^{m} \times B^{n}=\bigsqcup_{z \in B^{n}} \mathbb{R}^{m} \times\{z\}$. This foliation $\mathcal{F}_{\text {std }}$ has a transverse complex structure $J_{\text {std }}$ given by the complex structure of $\mathbb{C}^{n}$. We call $\left(\mathbb{R}^{m} \times B^{n}, \mathcal{F}_{\text {std }}, J_{\text {std }}\right)$ the standard transversely holomorphic foliation of $\mathbb{R}^{m} \times B^{n}$.

Example 2.8. The total space of a fiber bundle over a complex manifold has a transversely holomorphic Riemannian foliation whose leaves are fibers.

A fundamental result of Gómez Mont 23 is as follows:

Theorem 2.9. Any complex codimension $n$ transversely holomorphic foliation of dimension $m$ is locally isomorphic to $\left(\mathbb{R}^{m} \times B^{n}, \mathcal{F}_{\text {std }}, J_{\text {std }}\right)$.

By this result, transversely holomorphic foliations are described in terms of Haefliger 1-cocycles (see Section 3.2 for such a description).

The following is relevant in Sasakian geometry:

Definition 2.10. A triple $\left(\mathcal{F}, J, g_{\nu}\right)$ is called a transversely Kähler foliation of $M$ if

(i) $\left(\mathcal{F}, g_{\nu}\right)$ is a Riemannian foliation of $M$,

(ii) $(\mathcal{F}, J)$ is a transversely holomorphic foliation of $M$ and

(iii) the tensor field $\omega$ defined by $\omega(X, Y)=g_{\nu}(X, J Y)$ is antisymmetric and closed when regarded as a 2 -form on $M$ by the injection $\bigwedge^{2}\left(T^{*} M / T^{*} \mathcal{F}\right) \rightarrow$ $\wedge^{2} T^{*} M$.

We call the 2-form $\omega$ in (iii) the transverse Kähler form of $\left(\mathcal{F}, J, g_{\nu}\right)$. A transversely Kähler foliation of dimension one is called a transversely Kähler flow.

We will regard the transverse Kähler form $\omega$ of a transversely Kähler flow as a 2form on $M$ by the injection $\bigwedge^{2}\left(T^{*} M / T^{*} \mathcal{F}\right) \rightarrow \bigwedge^{2} T^{*} M$ throughout this article.

Boyer, Galicki and Nakamaye [9] proved that the Reeb flow of a Sasakian manifold has a transversely Kähler structure:

Lemma 2.11. The Reeb flow of a Sasakian manifold $(M, \eta, g)$ is transversely Kähler whose transverse Kähler form is $d \eta$.

2.3. $K$-contact structures. Let $\eta$ be a contact form on a closed manifold $M$ with a Riemannian metric $g$. We recall

Definition 2.12. $(M, \eta, g)$ is called a $K$-contact manifold if

(i) $g$ is preserved by the Reeb flow of $\eta$ and

(ii) there exists an almost complex structure $\Phi$ on ker $\eta$ such that $g(X, Y)=$ $d \eta(X, \Phi Y)$ for $X, Y$ in $C^{\infty}(\operatorname{ker} \eta)$.

A Sasakian structure is a $K$-contact structure (see Proposition 6.5 .12 of [8]). In particular, the Reeb flow of $\eta$ is Riemannian by condition (i). The converse is true by an observation of Yamazaki:

Proposition 2.13 (Yamazaki [45, Proposition 2.1]). A closed manifold with a contact form whose Reeb flow is a Riemannian flow admits a structure of the $K$ contact manifold. 
The characterization of Sasakian metrics in terms of $K$-contact structure in the last sentence of Section 6.4 of Blair [6] (see also [8, Proposition 6.5.14] is as follows:

Proposition 2.14. A $K$-contact structure is a Sasakian metric if the $C R$-structure (ker $\eta, \Phi)$ is integrable.

By Propositions 2.13 and 2.14 we get

Lemma 2.15. A pair of an integrable $C R$-structure $(H, \Phi)$ and a contact form $\eta$ determines a Sasakian metric if $H=\operatorname{ker} \eta$ and the Reeb flow of $\eta$ is Riemannian.

2.4. A characterization of Sasakian structures. We have the following characterization of Sasakian metrics in terms of transversely Kähler flows and contact forms:

Proposition 2.16. A pair of a transversely Kähler flow $\left(\mathcal{F}, J, g_{\nu}\right)$ and a contact form $\eta$ determines a Sasakian structure on $M$ if $d \eta=\omega$, where $\omega$ is the transverse Kähler form of $\left(\mathcal{F}, J, g_{\nu}\right)$.

Remark 2.17. It is well known that, conversely, the contact form and the underlying transversely Kähler structure of the Reeb flow of a Sasakian manifold satisfy the relation in Proposition 2.16.

Proof of Proposition 2.16. By Lemma 2.15, it suffices to show that a pair of a transversely Kähler flow $\left(\mathcal{F}, J, g_{\nu}\right)$ and a contact form $\eta$ such that $d \eta=\omega$ determines a pair of a $C R$-structure and a contact form which satisfy the conditions of Lemma 2.15. Let $H=\operatorname{ker} \eta$ and

$$
\pi: T M \rightarrow T M / T \mathcal{F}
$$

be the canonical projection. Letting $\Phi=\left(\left.\pi\right|_{H}\right)^{-1} \circ J \circ\left(\left.\pi\right|_{H}\right)$, we have a $C R$ structure $(H, \Phi)$ on $M$. Clearly the Reeb flow of $\eta$ is Riemannian by assumption. Thus the unique nontrivial part of the proof is the integrability of the $C R$-structure $(H, \Phi)$.

By $H=\operatorname{ker} \eta$, we get

$$
\frac{1}{2} \eta([X, Y]-[\Phi X, \Phi Y])=d \eta(X, Y)-d \eta(\Phi X, \Phi Y)
$$

for local sections $X$ and $Y$ of $H$. The right hand side is zero, because $d \eta$ is a transverse Kähler form, which is $\Phi$-invariant. It follows that $[\Phi X, \Phi Y]-[X, Y]$ is a local section of $H$ for any local sections $X$ and $Y$. This implies the first half of the integrability condition.

We will show that the Nijenhaus tensor

$$
N_{\Phi}(X, Y)=[\Phi X, \Phi Y]-\Phi([\Phi X, Y]+[X, \Phi Y])-[X, Y]
$$

vanishes for any local sections $X$ and $Y$ of $H$. Fix a point $x$ on $M$. Let $(\mathbb{R} \times$ $\left.B^{n}, \mathcal{F}_{\text {std }}, J_{\text {std }}\right)$ be the standard transversely holomorphic flow of $\mathbb{R} \times B^{n}$ mentioned in Example 2.7. By a theorem of Gómez Mont (Theorem 2.9), we have an open neighborhood $U_{x}$ of $x$ such that $\left(U_{x},\left.\mathcal{F}\right|_{U_{x}},\left.J\right|_{U_{x}}\right)$ is isomorphic to $\left(\mathbb{R} \times B^{n}, \mathcal{F}_{\text {std }}, J_{\text {std }}\right)$. We identify $U_{x}$ with $\mathbb{R} \times B^{n}$ by this isomorphism. By the product structure of $\mathbb{R} \times B^{n}$, we can decompose a vector field $X$ on $\mathbb{R} \times B^{n}$ as $X=X_{1}+X_{2}$, where $X_{1}$ is the $T \mathbb{R}$-component and $X_{2}$ is the $T B^{n}$-component. We take a section $X$ of $\left.H\right|_{U_{x}}$ so that the $T B^{n}$-component $X_{2}$ of $X$ is projectable to a linear vector field on $B^{n}$. 
We take another section $Y$ of $\left.H\right|_{U_{x}}$ in the same way. Clearly $X$ and $Y$ are basic vector fields. Let $H^{\prime}$ be the kernel of the differential map of the first projection $\mathbb{R} \times B^{n} \rightarrow \mathbb{R}$. Let $\Phi^{\prime}=\left(\left.\pi\right|_{H^{\prime}}\right)^{-1} \circ J \circ\left(\left.\pi\right|_{H^{\prime}}\right)$. Since the $T B^{n}$-components of $X$ and $Y$ are linear, we get $\left[X, \Phi^{\prime} Y\right] \in C^{\infty}(T \mathcal{F})$. Since $\left(\Phi-\Phi^{\prime}\right) Y \in C^{\infty}(T \mathcal{F})$ and $X$ is basic, we get $\left[X,\left(\Phi-\Phi^{\prime}\right) Y\right] \in C^{\infty}(T \mathcal{F})$. By $[X, \Phi Y]=\left[X,\left(\Phi-\Phi^{\prime}\right) Y\right]+\left[X, \Phi^{\prime} Y\right]$, it follows that $[X, \Phi Y] \in C^{\infty}(T \mathcal{F})$. Similarly, $[\Phi X, Y] \in C^{\infty}(T \mathcal{F})$. Thus we have

$$
\Phi([\Phi X, Y]+[X, \Phi Y])=0
$$

Hence we have

$$
N_{\Phi}(X, Y)=[\Phi X, \Phi Y]-[X, Y]
$$

Thus, by the last paragraph, $N_{\Phi}(X, Y)$ is a section of $\left.H\right|_{U_{x}}$. On the other hand, by the integrability of $J$, we have

$$
\pi\left(N_{\Phi}(X, Y)\right)=N_{J}(\pi(X), \pi(Y))=0 .
$$

Thus $N_{\Phi}(X, Y)$ is tangent to $\mathcal{F}$. Since $N_{\Phi}(X, Y)$ is a section of $\left.H\right|_{U_{x}}$ tangent to $\mathcal{F}$, we have $N_{\Phi}(X, Y)=0$.

The value of $N_{\Phi}(X, Y)$ at $x$ is determined only by vectors $X_{x}$ and $Y_{x}$. Thus the argument above shows $N_{\Phi}(X, Y)=0$ for any local section $X$ and $Y$ of $H$. Hence $(H, \Phi)$ is integrable.

2.5. Families of flows with transverse structures. Let $V$ be an open subset of $\mathbb{R}^{\ell}$. We define smooth families of flows with various transverse structures whose parameter space is $V$ to give the formalism to consider the deformation of flows. Note that our definition of smooth families of flows with transverse structures is equivalent to that of smooth families of deformation given in terms of families of 1cocycles used in Kodaira-Spencer theory [32] or deformation theory of transversely holomorphic foliations 22 .

Definition 2.18. $\quad$ (i) A smooth family of flows on $M$ over $V$ is a flow $\mathcal{F}^{\mathrm{amb}}$ on a smooth manifold $M \times V$ such that $M \times\{t\}$ is saturated by the leaves of $\mathcal{F}^{\mathrm{amb}}$. We denote the restriction of $\mathcal{F}^{\mathrm{amb}}$ to $M \times\{t\}$ by $\mathcal{F}^{t}$. We will denote such a family of flows on $M$ by $\left\{\mathcal{F}^{t}\right\}_{t \in V}$.

(ii) For a smooth family $\left\{\mathcal{F}^{t}\right\}_{t \in V}$ of flows on $M$, the kernel of the differential map of the second projection $\mathrm{pr}_{2}: T(M \times V) / T \mathcal{F}^{\mathrm{amb}} \rightarrow T V$ is called the family of normal bundles of $\left\{\mathcal{F}^{t}\right\}_{t \in V}$.

(iii) A smooth family of Riemannian flows on $M$ is a pair of a smooth family of flows $\mathcal{F}^{t}$ and a smooth Riemannian metric $g_{\nu}^{\text {amb }}$ on the family of normal bundles of $\left\{\mathcal{F}^{t}\right\}_{t \in V}$ such that $\left(\mathcal{F}^{t},\left.g^{\text {amb }}\right|_{M \times\{t\}}\right)$ is a Riemannian flow on $M$ for each $t$ in $V$.

(iv) A smooth family of transversely holomorphic flows on $M$ is a pair of a smooth family of flows $\left\{\mathcal{F}^{t}\right\}_{t \in V}$ and a complex structure $J^{\text {amb }}$ on the family of normal bundles of $\left\{\mathcal{F}^{t}\right\}_{t \in V}$ such that $\left(\mathcal{F}^{t},\left.J^{\mathrm{amb}}\right|_{M \times\{t\}}\right)$ is a transversely holomorphic flow on $M$ for each $t$ in $V$.

A smooth family of transversely Kähler flows on $M$ is similarly defined. 


\section{BASIC Euler CLASSES \\ OF TRANSVERSELY HOLOMORPHIC ISOMETRIC FLOWS}

3.1. Isometric flows. It is well known that the Reeb flow of a Sasakian manifold preserves the Riemannian metric (see [8, Proposition 6.5.6]. We recall

Definition 3.1. A Riemannian flow $\left(\mathcal{F}, g_{\nu}\right)$ on $M$ is called isometric if there exist a Riemannian metric $g$ on $M$ and a nowhere vanishing vector field $\xi$ tangent to $\mathcal{F}$ such that the flow generated by $\xi$ preserves $g$.

We will call $(g, \xi)$ in this definition a Killing pair on $\mathcal{F}$. A Riemannian metric $g$ on $M$ is called a Killing metric on $(M, \mathcal{F})$ if there exists a vector field $\xi$ such that $(g, \xi)$ is a Killing pair on $\mathcal{F}$. Note that the terminology "Killing pair" and "Killing metric" are not standard. We also recall

Definition 3.2. A foliated manifold $(M, \mathcal{F})$ is geometrically taut if there exists a Riemannian metric $g$ on $M$ such that every leaf of $\mathcal{F}$ is a minimal submanifold of $(M, g)$. Such $g$ is called a minimal metric on $(M, \mathcal{F})$.

Geometric tautness of Riemannian foliations was characterized in terms of basic cohomology by a theorem of Masa 34] in general, or a theorem of Molino and Sergiescu [37] in the case of flows. By the following lemma due to Carrière [12, the isometricity of oriented Riemannian flows is equivalent to the geometric tautness:

Lemma 3.3. Let $(M, \mathcal{F})$ be a closed manifold with an oriented Riemannian flow. Then a minimal metric $g$ is Killing if and only if $g$ is bundle-like.

Recall that a Riemannian metric $g$ on $(M, \mathcal{F})$ is called bundle-like if the metric induced on $T M / T \mathcal{F}$ from $g$ through the identification $T M / T \mathcal{F} \cong(T \mathcal{F})^{\perp}$ is a transverse metric of $\mathcal{F}$.

\section{2. $\mathcal{F}$-fibered Hermitian vector bundles and basic Dolbeault cohomol-} ogy. El Kacimi Alaoui [19] defined an $\mathcal{F}$-fibered Hermitian vector bundle on $(M, \mathcal{F})$ by a Hermitian vector bundle $(E, h)$ on $M$ such that the Chern connection is basic and $\nabla_{X}^{\otimes} h=0$ for any $X$ in $C^{\infty}(T \mathcal{F})$, where $\nabla^{\otimes}$ is the connection induced on $E^{*} \otimes \bar{E}^{*}$. We will give another equivalent definition of $\mathcal{F}$-fibered Hermitian vector bundles on foliated manifolds $(M, \mathcal{F})$ which is convenient in this article. We use $\mathbb{C}$ as the coefficient field of differential forms throughout Section 3.2

Let $(\mathcal{F}, J)$ be a transversely holomorphic foliation of real dimension $m$ and complex codimension $n$ on a closed manifold $M$. Let $B^{n}$ be the unit disk in $\mathbb{C}^{n}$. Let $\left(\mathcal{F}_{\text {std }}, J_{\text {std }}\right)$ be the standard transversely holomorphic foliation of $\mathbb{R}^{m} \times B^{n}$ mentioned in Example 2.7. At each point $x$ on $M$, we have an open neighborhood $U$ of $x$ such that $\left(U,\left.\mathcal{F}\right|_{U},\left.J\right|_{U}\right)$ is isomorphic to $\left(\mathbb{R}^{m} \times B^{n}, \mathcal{F}_{\text {std }}, J_{\text {std }}\right)$ by a theorem of Gómez Mont (Theorem 2.9). So we take a covering $\left\{U_{j}\right\}$ of $M$ such that $\left(U_{j},\left.\mathcal{F}\right|_{U_{j}},\left.J\right|_{U_{j}}\right)$ is isomorphic to $\left(\mathbb{R}^{m} \times B_{j}^{n}, \mathcal{F}_{\text {std }}, J_{\text {std }}\right)$ as transversely holomorphic foliations, where $B_{j}^{n}$ is the unit ball in $\mathbb{C}^{n}$. We denote the composite

$$
U_{j} \longrightarrow \mathbb{R}^{m} \times B_{j}^{n} \longrightarrow B_{j}^{n}
$$

by $\phi_{j}$, where the second map is the second projection.

Definition 3.4. A Hermitian vector bundle $\left(E, h_{E}\right)$ on $M$ is called $\mathcal{F}$-fibered if there exists a Hermitian vector bundle $\left(E_{j}, h_{j}\right)$ on $B_{j}^{n}$ such that $\left(\left.E\right|_{U_{j}},\left.h_{E}\right|_{U_{j}}\right)=$ $\left(\phi_{j}^{*} E_{j}, \phi_{j}^{*} h_{j}\right)$ for every $j$. An $\mathcal{F}$-fibered Hermitian vector bundle is holomorphic if 
such $\left(E_{j}, h_{j}\right)$ can be taken so that transition functions $\left.\left.E_{j}\right|_{\phi_{j}\left(U_{j} \cap U_{j^{\prime}}\right)} \rightarrow E_{j^{\prime}}\right|_{\phi_{j^{\prime}}\left(U_{j} \cap U_{j^{\prime}}\right)}$ are holomorphic on $\phi_{j}\left(U_{j} \cap U_{j^{\prime}}\right)$ for each $j$ and $j^{\prime}$ satisfying $U_{j} \cap U_{j^{\prime}} \neq \emptyset$.

This definition of $\mathcal{F}$-fibered Hermitian vector bundles is equivalent to the original definition of El Kacimi Alaoui as follows:

Lemma 3.5. For a Hermitian vector bundle $(E, h)$, the Chern connection is basic and $\nabla_{X}^{\otimes} h=0$ for any $X$ in $C^{\infty}(T \mathcal{F})$ if and only if $(E, h)$ is $\mathcal{F}$-fibered in the meaning of Definition 3.4.

Proof. Let $\pi: P \rightarrow M$ be the $\mathrm{GL}(r, \mathbb{C})$-principal bundle of $E$, where $r$ is the rank of $E$. By [31, Example 5.2 on p. 76], we can identify $h$ with a function $f: P \rightarrow$ $\mathbb{C}^{r *} \otimes{\overline{\mathbb{C}^{r}}}^{*}$ such that $f(u g)=\rho\left(g^{-1}\right) f(u)$ for any $g$ in $\operatorname{GL}(r, \mathbb{C})$ and any $u$ in $P$, where $\rho: \mathrm{GL}(r, \mathbb{C}) \rightarrow \mathrm{GL}\left(\mathbb{C}^{r *} \otimes \overline{\mathbb{C}}^{*}\right)$ is the canonical action. By 31, Lemma on p. 115], we have $\nabla_{X}^{\otimes} h(\pi(u))=u(\tilde{X} f)$ for a point $u$ on $P$, and a vector field $X$, where $u$ is regarded as an isomorphism $\mathbb{C}^{r *} \otimes \overline{\mathbb{C}}^{*} \rightarrow E_{\pi(u)}$ and $\widetilde{X}$ is the horizontal lift of $X$. The formula implies the equivalence.

Example 3.6. $\bigwedge^{k}\left(T^{*} M / T^{*} \mathcal{F}\right)^{1,0} \rightarrow M$ is a holomorphic $\mathcal{F}$-fibered vector bundle on $(M, \mathcal{F})$.

A foliated manifold has a cohomology called basic cohomology, which can be regarded as the de Rham cohomology of the leaf space in a sense. We refer to Masa [34] or El Kacimi Alaoui and Hector [20] for the basic cohomology of Riemannian foliations. We will recall the definition of a Dolbeault version of basic cohomology for holomorphic $\mathcal{F}$-fibered vector bundles $E$.

Definition 3.7. A differential form $\alpha$ on $M$ with values in $E$ is a basic $(p, q)$-form on $(M, \mathcal{F})$ with values in $E$ if there exists a $(p, q)$-form $\alpha_{j}$ on $B_{j}^{n}$ with values in $E_{j}$ such that $\left.\alpha\right|_{U_{j}}=\phi_{j}^{*} \alpha_{j}$ for every $j$. We denote the space of basic $(p, q)$-forms on $(M, \mathcal{F}, J)$ with values in $E$ by $\Omega_{b}^{p, q}(M / \mathcal{F}, E)$.

This notion is also independent of the foliated atlas $\left\{U_{j}\right\}$. In the case where $E$ is the trivial bundle of rank 1 , it is easy to see that the space of basic $k$-form $\Omega_{b}^{k}(M / \mathcal{F})$ is given by

$$
\Omega_{b}^{k}(M / \mathcal{F})=\left\{\alpha \in \Omega^{k}(M) \mid \iota_{X} \alpha=0, \iota_{X} d \alpha=0, \forall X \in C^{\infty}(T \mathcal{F})\right\} .
$$

We also recall

Definition 3.8. A section $s$ of $E$ is basic if there exists a section $s_{j}$ of $E_{j}$ such that $\left.s\right|_{U_{j}}=\phi_{j}^{*} s_{j}$.

Let $\left(E, h_{E}\right)$ be a holomorphic $\mathcal{F}$-fibered Hermitian vector bundle on $(M, \mathcal{F})$.

Definition 3.9. The basic Dolbeault operator

$$
\bar{\partial}_{E}: \Omega_{b}^{p, q}(M / \mathcal{F}, E) \rightarrow \Omega_{b}^{p, q+1}(M / \mathcal{F}, E)
$$

is defined by

$$
\left.\left(\bar{\partial}_{E} \alpha\right)\right|_{U_{j}}=\phi_{j}^{*}\left(\sum_{i}\left(\bar{\partial}_{B^{n}} \beta_{i}\right) \otimes s_{i}\right)
$$

for $\alpha$ in $\Omega_{b}^{p, q}(M / \mathcal{F}, E)$, where $s_{i}$ is a local holomorphic section of $E_{j}$, and $\left.\alpha\right|_{U_{j}}$ is written as $\left.\alpha\right|_{U_{j}}=\phi_{j}^{*}\left(\sum_{i} \beta_{i} \otimes s_{i}\right)$, and $\bar{\partial}_{B^{n}}$ is the Dolbeault operator on $B^{n}$. 
Then $\left(\Omega_{b}^{p, \bullet}(M / \mathcal{F}, E), \bar{\partial}_{E}\right)$ is a differential complex.

Definition 3.10. The $p$-th basic Dolbeault cohomology $H_{b}^{p, \bullet}(M / \mathcal{F}, E)$ of $(\mathcal{F}, J)$ with values in $E$ is defined by

$$
H_{b}^{p, \bullet}(M / \mathcal{F}, E)=H^{\bullet}\left(\Omega_{b}^{p, \bullet}(M / \mathcal{F}, E), \bar{\partial}_{E}\right) .
$$

Remark 3.11. If the leaves of $\mathcal{F}$ are not closed, the sheaf of $(p, q)$-forms with values in $E$ may not be acyclic. Hence we may not have an isomorphism $H_{b}^{p, q}(M / \mathcal{F}, E)$ between $H^{q}\left(M, \Omega_{b}^{p} \otimes E\right)$, where $\Omega_{b}^{p}$ is the sheaf of holomorphic basic $p$-forms on $(M, \mathcal{F}, J)$. Here the situation is different from the case of complex manifolds or complex orbifolds, where we always have $H_{b}^{p, q}(M / \mathcal{F}, E) \cong H^{q}\left(M, \Omega_{b}^{p} \otimes E\right)$.

3.3. Basic Euler classes of isometric flows. Let $\left(\mathcal{F}, g_{\nu}\right)$ be an isometric Riemannian flow on a closed smooth manifold $M$. We recall the definition of the basic Euler classes of $(M, \mathcal{F})$ due to Saralegui [43. We denote the space of basic $k$-forms on $(M, \mathcal{F})$ of a complex coefficient by $\Omega_{b}^{k}(M / \mathcal{F})_{\mathbb{C}}$. We denote the basic cohomology of $(M, \mathcal{F})$ by $H_{b}^{\bullet}(M / \mathcal{F})$.

Definition 3.12 (Saralegui 43 ). Let $(g, \xi)$ be a Killing pair on $\mathcal{F}$. We define a 1 -form $\eta$ on $M$ by $\eta(Y)=g(\xi, Y)$. Then $d \eta$ is a basic 2 -form on $(M, \mathcal{F})$. The basic Euler class of $\mathcal{F}$ is defined by $\mathbb{R}^{\times}[d \eta]$ in $H_{b}^{2}(M / \mathcal{F})$ up to multiplication of nonzero real numbers.

Saralegui 43 proved that the basic Euler class of $\mathcal{F}$ depends only on the smooth type of the flow $\mathcal{F}$ (see Royo Prieto [4] for the generalization of the definition of basic Euler classes for Riemannian flows).

Example 3.13. If $\mathcal{F}$ is an isometric flow defined by fibers of a circle bundle, the basic cohomology of $\mathcal{F}$ coincides with the de Rham cohomology of the base manifold. In this case, the basic Euler class of $\mathcal{F}$ coincides with the Euler class of the circle bundle up to multiplication of real numbers.

3.4. The (0,2)-component of the basic Euler class of transversely holomorphic flows. Let $M$ be a closed smooth manifold. Let $\left(\mathcal{F}, g_{\nu}, J\right)$ be a transversely holomorphic Riemannian flow on $M$. We assume that $\left(\mathcal{F}, g_{\nu}\right)$ is isometric with a Killing pair $(g, \xi)$. Let $\eta$ be the characteristic form of $(M, \mathcal{F}, g)$ defined by $\eta(X)=g(\xi, X)$. Then the basic cohomology class of $d \eta$ is the basic Euler class of $(M, \mathcal{F})$ by Definition 3.12 , Note that the $(0,2)$-component $(d \eta)^{0,2}$ of $d \eta$ is $\bar{\partial}$-closed, because $\bar{\partial}(d \eta)^{0,2}=(d d \eta)^{0,3}=0$.

Definition 3.14. We define the (0,2)-component of the basic Euler class of $(\mathcal{F}, g, J)$ by $\mathbb{R}^{\times}\left[(d \eta)^{0,2}\right]$ in $H_{b}^{0,2}(M / \mathcal{F})$ up to multiplication of nonzero real numbers. If the $(0,2)$-component of the basic Euler class of $(\mathcal{F}, g, J)$ is trivial, we say the basic Euler class of $(\mathcal{F}, g, J)$ is of degree $(1,1)$.

We will show the well definedness of the $(0,2)$-component of the basic Euler class in the following. We will use the leafwise cohomology of $(M, \mathcal{F})$. Let $\Omega^{k}(\mathcal{F})_{\mathbb{C}}=$ $C^{\infty}\left(\bigwedge^{k} T^{*} \mathcal{F}\right) \otimes \mathbb{C}$. The differential on each leaf of $\mathcal{F}$ yields the leafwise differential

$$
d_{\mathcal{F}}: \Omega^{k}(\mathcal{F})_{\mathbb{C}} \rightarrow \Omega^{k+1}(\mathcal{F})_{\mathbb{C}}
$$

The cohomology of the differential complex $\left(\Omega^{\bullet}(\mathcal{F}), d_{\mathcal{F}}\right)$ is called the leafwise cohomology of $(M, \mathcal{F})$. We denote it by $H^{\bullet}(\mathcal{F})$. 
Remark 3.15. The leafwise cohomology may be of infinite dimension even for linear flows on tori (see El Kacimi Alaoui [19]).

The restriction $\left.\eta\right|_{T \mathcal{F}}$ of a characteristic form $\eta$ of $\mathcal{F}$ to $T \mathcal{F}$ satisfies $d_{\mathcal{F}}\left(\left.\eta\right|_{T \mathcal{F}}\right)=0$, thus it determines a leafwise cohomology class $\left[\left.\eta\right|_{T \mathcal{F}}\right]$ in $H^{1}(\mathcal{F})$. We get

Lemma 3.16. Let $\eta_{j}$ be the characteristic forms of $\left(M, \mathcal{F}, g_{j}\right)$ for a Killing metric $g_{j}$ for $j=1$ and 2 . Then there exists a nonzero real number $r$ such that

$$
\left[\left.\eta_{1}\right|_{T \mathcal{F}}\right]=r\left[\left.\eta_{2}\right|_{T \mathcal{F}}\right]
$$

in $H^{1}(\mathcal{F})$.

Proof. $H^{1}(\mathcal{F})$ can be identified with the $(0,1)$-th $E_{1}$-term $E_{1}^{0,1}(\mathcal{F})$ of the spectral sequence canonically associated to $\mathcal{F}$ (see Kamber and Tondeur 30]). We can identify the $(0,1)$-th $E_{2}$-term $E_{2}^{0,1}(\mathcal{F})$ with a subspace of $E_{1}^{0,1}(\mathcal{F})$ consisting of $d_{1}$-closed elements. By [30, Corollary 4.7], we get $\operatorname{dim} E_{2}^{0,1}(\mathcal{F})=1$, and $E_{2}^{0,1}(\mathcal{F})$ is generated by the leafwise cohomology class of the characteristic form of any minimal metric under these identifications. Since $g_{j}$ is minimal by Lemma 3.3 for $j=1$ and 2, we get nonzero real number $r$ such that $\left[\left.\eta_{1}\right|_{T \mathcal{F}}\right]=r\left[\left.\eta_{2}\right|_{T \mathcal{F}}\right]$ in $H^{1}(\mathcal{F})$.

Let $\operatorname{Diff}(M, \mathcal{F})$ be the subgroup of $\operatorname{Diff}(M)$ consisting of diffeomorphisms which map each leaf of $\mathcal{F}$ to itself. Let $\operatorname{Diff}_{0}(M, \mathcal{F})$ be the identity component of $\operatorname{Diff}(M, \mathcal{F})$ with respect to the Fréchet topology.

Lemma 3.17. Let $\eta_{j}$ be the characteristic form of $\left(M, \mathcal{F}, g_{j}\right)$ for a Killing metric $g_{j}$ for $j=1$ and 2 . There exists $r$ in $\mathbb{R}$ and $f$ in $\operatorname{Diff}_{0}(M, \mathcal{F})$ such that

$$
\left.\eta_{1}\right|_{T \mathcal{F}}=r f^{*}\left(\left.\eta_{2}\right|_{T \mathcal{F}}\right) \text {. }
$$

Proof. By Lemma 3.16, we have a real number $r$ such that $\left[\left.\eta_{1}\right|_{T \mathcal{F}}\right]=r\left[\left.\eta_{2}\right|_{T \mathcal{F}}\right]$ in $H^{1}(\mathcal{F})$. By the leafwise version of Moser's argument (see Hector, Macias and Saralegui [29]), we have a diffeomorphism $f$ of $M$ in $\operatorname{Diff}_{0}(M, \mathcal{F})$ such that $\left.\eta_{1}\right|_{T \mathcal{F}}=$ $r f^{*}\left(\left.\eta_{2}\right|_{T \mathcal{F}}\right)$.

Lemma 3.18. The $(0,2)$-component of the basic Euler class is well defined for $(\mathcal{F}, J)$ up to multiplication of nonzero real numbers.

Proof. Let $\left(g_{1}, \xi_{1}\right)$ and $\left(g_{2}, \xi_{2}\right)$ be two Killing pairs on $(M, \mathcal{F})$. Let $\eta_{j}$ be the characteristic form of $\left(M, \mathcal{F}, g_{j}\right)$ for $j=1$ and 2. By Lemma 3.17, there exist a real number $r$ and a diffeomorphism $f$ in $\operatorname{Diff}_{0}(M, \mathcal{F})$ such that $\left.\eta_{1}\right|_{T \mathcal{F}}=r f^{*}\left(\left.\eta_{2}\right|_{T \mathcal{F}}\right)$. Thus $f_{*} \xi_{1}=r \xi_{2}$. Here $\eta_{1}-r f^{*} \eta_{2}$ is a basic 1 -form by $\left.\left(\eta_{1}-r f^{*} \eta_{2}\right)\right|_{T \mathcal{F}}=0$ and $\mathcal{L}_{\xi_{1}}\left(\eta_{1}-r f^{*} \eta_{2}\right)=0$. Hence we get $\left[\left(d \eta_{1}\right)^{0,2}\right]=r f^{*}\left[\left(d \eta_{2}\right)^{0,2}\right]$ by

$$
\bar{\partial}\left(\left(\eta_{1}-r f^{*} \eta_{2}\right)^{0,1}\right)=\left(d \eta_{1}\right)^{0,2}-r f^{*}\left(d \eta_{2}\right)^{0,2} \text {. }
$$

Since $f$ belongs to $\operatorname{Diff}_{0}(M, \mathcal{F})$, Proposition II.1.c of El Kacimi Alaoui 18 implies that $f$ trivially acts on the leafwise cohomology. Then $f^{*}\left[\left(d \eta_{2}\right)^{0,2}\right]=\left[\left(d \eta_{2}\right)^{0,2}\right]$. Thus we get $\left[\left(d \eta_{1}\right)^{0,2}\right]=r\left[\left(d \eta_{2}\right)^{0,2}\right]$, which concludes the proof.

By Lemma 2.11, the underlying transversely holomorphic Riemannian flow of a Sasakian manifold $(M, g, \eta)$ is transversely Kähler with transverse Kähler form $d \eta$. Since a transverse Kähler form is always of degree $(1,1)$, we have

Lemma 3.19. The basic Euler classes of the underlying transversely holomorphic isometric Riemannian flows of Sasakian manifolds are of degree $(1,1)$. 


\section{EXISTEnCE OF EXTEnsions of SASAKIAN METRICS}

4.1. A differential operator $D$. Let $\left(M, \mathcal{F}, J, g_{\nu}\right)$ be a closed manifold with a transversely holomorphic Riemannian flow. We extend $g_{\nu}$ to a $J$-invariant Hermitian metric on $(T M / T \mathcal{F}) \otimes \mathbb{C}$. We introduce a triple grading of $\Omega^{\bullet}(M)_{\mathbb{C}}=$ $\Omega^{\bullet}(M) \otimes \mathbb{C}$, which is known for researchers of CR geometry. The triple grading is the complex version of the double grading mentioned in Section 4.2, which is well known to researchers of foliation theory. Then we will introduce a differential operator $D$ to be used later in the proof of Theorem 1.1.

Let $(g, \xi)$ be a Killing pair on $\mathcal{F}$ such that the transverse metric on $T M / T \mathcal{F}$ induced from $g$ is equal to $g_{\nu}$. Let $\eta$ be the characteristic form of $(M, \mathcal{F}, g)$ defined by $\eta(X)=g(\xi, X)$. Let $H=\operatorname{ker} \eta$ and denote the orthogonal projection $T M \rightarrow H$ by $\pi$. We have a $C R$-structure $(H, \Phi)$ defined by $\Phi=\left(\left.\pi\right|_{H}\right)^{-1} \circ J \circ\left(\left.\pi\right|_{H}\right)$. Let $H^{0,1}$ (resp. $H^{1,0}$ ) be the vector subbundle of $H \otimes \mathbb{C}$ whose fibers are the $(-i)$-eigenspaces (resp. $i$-eigenspaces) of $\Phi$.

The coefficient ring of differential forms is $\mathbb{C}$ in Section 4.1. By the decomposition $T M \otimes \mathbb{C}=H^{1,0} \oplus H^{0,1} \oplus(T \mathcal{F} \otimes \mathbb{C})$, we define a triple grading $\Omega^{\bullet}(M)_{\mathbb{C}}=\Omega_{h, j, k}$, where

$$
\Omega_{h, j, k}=C^{\infty}\left(\bigwedge^{h}\left(H^{1,0}\right)^{*} \otimes \bigwedge^{j}\left(H^{0,1}\right)^{*} \otimes \bigwedge^{k}(T \mathcal{F} \otimes \mathbb{C})^{*}\right) .
$$

By the integrability of $\mathcal{F}$, we can decompose the differential $d$ as

$$
d=d_{0,0,1}+d_{0,1,0}+d_{1,0,0}+d_{0,2,-1}+d_{1,1,-1}+d_{2,0,-1},
$$

where $d_{h^{\prime}, j^{\prime}, k^{\prime}}$ is the $\left(\Omega_{h+h^{\prime}, j+j^{\prime}, k+k^{\prime}}\right)$-component of $d$ on $\Omega_{h, j, k}$. Note that the space of basic $(p, q)$-forms is embedded to $\Omega_{p, q, 0}$. We define a differential operator

$$
D=d-d_{1,1,-1} \text {. }
$$

Let $D^{*}$ be the formal adjoint of $D$ with respect to the inner product on $\Omega^{\bullet}(M)_{\mathbb{C}}$ defined by $\left\langle\alpha_{1}, \alpha_{2}\right\rangle=\int_{M} \alpha_{1} \wedge{ }_{*} \alpha_{2}$ for $\alpha_{1}$ and $\alpha_{2}$ in $\Omega^{k}(M)_{\mathbb{C}}$. Let

$$
\Delta_{D}=D D^{*}+D^{*} D
$$

and

$\mathbf{H}_{D}^{k}=\operatorname{ker}\left\{\Delta_{D}: \Omega^{k}(M)_{\mathbb{C}} \rightarrow \Omega^{k}(M)_{\mathbb{C}}\right\}, \quad \mathbf{H}^{k}=\operatorname{ker}\left\{\Delta: \Omega^{k}(M)_{\mathbb{C}} \rightarrow \Omega^{k}(M)_{\mathbb{C}}\right\}$, where $\Delta=d d^{*}+d^{*} d$.

We collect fundamental properties of $D$ as follows:

Lemma 4.1. (i) $\Delta_{D}$ is a self-adjoint strongly elliptic operator.

(ii) $D^{*}=-\bar{*} D \bar{*}$.

(iii) Any d-closed form is D-closed, and any $d^{*}$-closed form is $D^{*}$-closed.

(iv) $\mathbf{H}^{k} \subseteq \mathbf{H}_{D}^{k}$.

(v) $\mathbf{H}_{D}^{k}=\operatorname{ker} D \cap \operatorname{ker} D^{*}$.

Proof. For $\alpha$ in $\Omega_{h, j, 1}$, we have

$$
\begin{aligned}
d_{1,1,-1} & \alpha\left(X_{1}, \ldots, X_{h+1}, Y_{1}, \ldots, Y_{j+1}\right) \\
& =\sum_{s<t}(-1)^{s(h+1+t)} \alpha\left(\left[X_{s}, Y_{t}\right], X_{1}, \ldots, \widehat{X}_{s}, \ldots, X_{h+1}, Y_{1}, \ldots, \widehat{Y}_{t}, \ldots, Y_{j+1}\right)
\end{aligned}
$$

for $X_{1}, \ldots, X_{h+1}$ in $C^{\infty}\left(H^{1,0}\right)$ and $Y_{1}, \ldots, Y_{j+1}$ in $C^{\infty}\left(H^{0,1}\right)$. Thus $d_{1,1,-1}$ satisfies $d_{1,1,-1}(f \alpha)=f d_{1,1,-1} \alpha$ for any smooth function $f$ on $M$, which means that $d_{1,1,-1}$ 
is a differential operator of degree 0 . This implies that the symbol $\Delta_{D}$ is equal to the symbol $\Delta$. Thus (i) is proved. We have (ii) by integrating both sides of

$$
d\left(\alpha_{1} \wedge \bar{*} \alpha_{2}\right)=D \alpha_{1} \wedge \bar{*} \alpha_{2}+(-1)^{k} \alpha_{1} \wedge D \bar{*} \alpha_{2}
$$

on $M$ for $\alpha_{1}$ in $\Omega_{h, j, k}$ and $\alpha_{2}$ in $\Omega_{h-1, j, k}, \Omega_{h, j-1, k}$ or $\Omega_{h, j, k-1}$. Note that $\bar{*}\left(\Omega_{j, k, h}\right) \subseteq$ $\Omega_{n-j, n-k, 1-h}$, where $2 n$ is the real codimension of $\mathcal{F}$. By $\bar{*}\left(\Omega_{j, k, h}\right) \subseteq \Omega_{n-j, n-k, 1-h}$ and the definition of $D$, we get (iv). By the definition of $\Delta_{D}$, it follows that ker $D \cap \operatorname{ker} D^{*} \subseteq \mathbf{H}_{D}^{k}$. Conversely $\mathbf{H}_{D}^{k} \subseteq \operatorname{ker} D \cap \operatorname{ker} D^{*}$ by the equality $\left\langle\Delta_{D} \alpha, \alpha\right\rangle=$ $\langle D \alpha, D \alpha\rangle+\left\langle D^{*} \alpha, D^{*} \alpha\right\rangle$. Thus (v) is proved.

Lemma 4.2. Assume that $(\eta, g)$ is a Sasakian metric on $M$. Then we have an equality

$$
\mathbf{H}_{D}^{1}=\mathbf{H}^{1} \oplus \mathbb{C} \eta
$$

in $\Omega^{1}(M)_{\mathbb{C}}$

Proof. We show $\mathbf{H}^{1} \oplus \mathbb{C} \eta \subseteq \mathbf{H}_{D}^{1}$. Since $d \eta$ is a basic $(1,1)$-form on $(M, \mathcal{F})$, we have $d \eta=d_{1,1,-1} \eta$. Thus

$$
D \eta=0 .
$$

Since $\bar{*} \eta$ is a basic volume form on $(M, \mathcal{F})$ by Equation 2.8 of Kamber and Tondeur [30, we have $d \bar{*} \eta=0$. It follows that

$$
D^{*} \eta=0 .
$$

Hence $\eta$ is $\Delta_{D}$-harmonic. We get $\mathbf{H}^{1} \subseteq \mathbf{H}_{D}^{1}$ by Lemma 4.1 (iv). Since $\eta$ is not $d$-closed, $\eta$ is not contained in $\mathbf{H}^{1}$. Thus $\mathbf{H}^{1} \oplus \mathbb{C} \eta \subseteq \mathbf{H}_{D}^{1}$.

We show $\mathbf{H}_{D}^{1} \subseteq \mathbf{H}^{1} \oplus \mathbb{C} \eta$. Let $\alpha$ be a $\Delta_{D}$-harmonic 1-form. By Lemma $4.1(\mathrm{v})$, we get $D \alpha=0$ and $D^{*} \alpha=0$. Let $\left\{\varphi_{u}\right\}_{u \in \mathbb{R}}$ be the flow generated by the Reeb vector field $\xi$ of $\alpha$. Since $\varphi_{u}$ preserves any of $J, H$ and $g$, we have

$$
\Delta_{D} \varphi_{u}^{*}=\varphi_{u}^{*} \Delta_{D}
$$

Thus $\varphi_{u}^{*}$ preserves $\mathbf{H}_{D}^{1}$. Since $\iota_{\xi}$ is the zero map on $\Omega_{j, k, 0}$, we have $\iota_{\xi} d_{1,1,-1} \alpha=0$. It follows that $\iota_{\xi} d \alpha=\iota_{\xi} D \alpha=0$. Then

$$
\mathcal{L}_{\xi} \alpha=d(\alpha(\xi))
$$

By (10), we have

$$
\varphi_{u}^{*} \alpha-\alpha=\left.\int_{0}^{u} \frac{d}{d r}\right|_{r=s}\left(\varphi_{r}^{*} \alpha\right) d s=\int_{0}^{u} \varphi_{s}^{*}\left(\mathcal{L}_{\xi} \alpha\right) d s=d\left(\int_{0}^{u} \varphi_{s}^{*} \alpha(\xi) d s\right),
$$

which implies that $\varphi_{u}^{*} \alpha-\alpha$ is an exact 1 -form. Since $d_{1,1,-1} \bar{*}\left(\varphi_{u}^{*} \alpha-\alpha\right)=0$ because of the degree, we have

$$
d^{*}\left(\varphi_{u}^{*} \alpha-\alpha\right)=D^{*}\left(\varphi_{u}^{*} \alpha-\alpha\right)=0 .
$$

By the Hodge decomposition of $\Omega^{1}(M)_{\mathbb{C}}$, a $d$-exact and $d^{*}$-closed form is zero. Hence

$$
\varphi_{u}^{*} \alpha-\alpha=0
$$

Thus $\alpha$ is invariant under the flow generated by $\xi$, which implies $\alpha(\xi)$ is a leafwise constant function on $(M, \mathcal{F})$. Letting $\beta=\alpha-\alpha(\xi) \eta$ and $f=\alpha(\xi)$, we decompose $\alpha$ as

$$
\alpha=\beta+f \eta \text {. }
$$


Here $\beta$ is a basic 1 -form on $(M, \mathcal{F})$, because $\varphi_{u}^{*} \beta-\beta=0$ and satisfies $\beta(\xi)=0$. By differentiating both sides of (11) and operating $\iota_{\xi}$, we have

$$
d f=0 .
$$

Thus $f$ is a constant. Since $D=d$ on $\Omega_{j, k, 0}$ by definition, we have

$$
0=D \alpha=D(\beta+f \eta)=d \beta+f D \eta .
$$

By (8), we have $d \beta=0$. We have

$$
0=D^{*} \alpha=-\bar{*} D \bar{*} \beta+f D^{*} \eta=-\bar{*} d \bar{*} \beta+f D^{*} \eta,
$$

because $D=d$ on $\bigoplus_{j+k=2 n-1} \Omega_{j, k, 1}$, where $2 n$ is the real codimension of $\mathcal{F}$. By (9), we have $d^{*} \beta=0$. Hence $\beta$ is a harmonic 1-form. Thus we get $\mathbf{H}_{D}^{1} \subseteq \mathbf{H}^{1} \oplus \mathbb{C} \eta$.

4.2. Mean curvature forms of Riemannian flows. We will make an introduction to the description of mean curvature forms of Riemannian foliations by Álvarez López [3] in the special case of Riemannian flows to prove a preliminary result necessary in the proof of Theorem 1.1 in the next section. Let $(M, \mathcal{F})$ be a flow. Let $g$ be a Riemannian metric on $M$. We recall

Definition 4.3. The mean curvature form of $(M, \mathcal{F}, g)$ is a 1 -form $\kappa$ on $M$ defined by

$$
\kappa(X)=g\left(\xi, \nabla_{\xi} X\right)
$$

for $X$ in $C^{\infty}(T M)$, where $\nabla$ is the Levi-Civita connection of $(M, g)$ and $\xi$ is a vector field tangent to $\mathcal{F}$ such that $g(\xi, \xi)=1$.

For a point $x$ on $M$, the 1 -form $\kappa_{x}$ is the mean curvature form of the leaf of $\mathcal{F}$ which goes through $x$. Thus $\kappa=0$ if and only if every leaf of $\mathcal{F}$ is a geodesic with respect to $g$ under the length parametrization.

Let $\eta$ be the characteristic form of $(M, \mathcal{F}, g)$, which is defined by $\eta(X)=g(\xi, X)$. Then Rummler's formula (see the second formula in the proof of [42, Proposition 1] or [11, Lemma 10.5.6] gives

$$
\kappa(X)=d \eta(\xi, X)
$$

for $X$ in $C^{\infty}(T M)$. Thus $\kappa$ is determined only by $\left.g\right|_{T \mathcal{F} \otimes T \mathcal{F}}$ and the orthogonal plane field $(T \mathcal{F})^{\perp}$, because $\eta$ is clearly determined only by $\left.g\right|_{T \mathcal{F} \otimes T \mathcal{F}}$ and $(T \mathcal{F})^{\perp}$.

In the case where $\mathcal{F}$ is Riemannian and $g$ is bundle-like, Álvarez López [3] calculated the change of $\kappa$ when $\left.g\right|_{T \mathcal{F} \otimes T \mathcal{F}}$ and $(T \mathcal{F})^{\perp}$ are changed. To state his calculation, we introduce a well-known double grading of $\Omega^{\bullet}(M)$. In Section 4.2, we consider only real differential forms. Let $Q=(T \mathcal{F})^{\perp}$ and

$$
A_{j, k}=C^{\infty}\left(\bigwedge^{j} Q^{*} \otimes \bigwedge^{k} T^{*} \mathcal{F}\right) .
$$

Then $\Omega^{\bullet}(M)$ is decomposed as

$$
\Omega^{\bullet}(M)=\bigoplus_{j, k} A_{j, k} .
$$

By the integrability of $T \mathcal{F}$, we see that the differential $d$ is decomposed as

$$
d=d_{0,1}+d_{1,0}+d_{2,-1},
$$


where the subscripts correspond to the double grading of $\Omega^{\bullet}(M)$. Since $g$ is bundlelike, the formal adjoint $d^{*}$ of $d$ is decomposed as

$$
d^{*}=d_{0,-1}^{*}+d_{-1,0}^{*}+d_{-2,1}^{*}
$$

by the double grading of $\Omega^{\bullet}(M)$ in a similar way.

Let $g_{1}$ be another bundle-like metric on $(M, \mathcal{F})$ with characteristic form $\eta_{1}$. Let $\kappa_{1}$ be the mean curvature form of $\left(M, \mathcal{F}, g_{1}\right)$. Let $Q_{1}$ be the orthogonal plane field of $T \mathcal{F}$ with respect to $g_{1}$. If $Q=Q_{1}$, then there exists a smooth function $f$ on $M$ such that $\eta=e^{f} \eta_{1}$. Then, by Rummler's formula (12), we get

Proposition 4.4 (Álvarez López [3, Eq. 5.3]). If $Q=Q_{1}$, then

$$
\kappa_{1}-\kappa=d_{1,0} f
$$

for some $f$ in $A_{0,0}$. Conversely, for any $f$ in $A_{0,0}$, there exists a bundle-like metric $g_{1}$ on $(M, \mathcal{F})$ such that $Q_{1}=Q$ and (15) is satisfied. Moreover, if $f$ is $C^{\infty}$-close to 0 , then we can take such $g_{1}$ so that $g_{1}$ is $C^{\infty}$-close to $g$.

Álvarez López calculated the effect of the change of $Q_{1}$ to $\kappa_{1}$ in terms of $d_{0,-1}^{*}$ as follows:

Proposition 4.5 (Álvarez López [3, Proposition 4.3]). If $\left.g_{1}\right|_{T \mathcal{F} \otimes T \mathcal{F}}=\left.g\right|_{T \mathcal{F} \otimes T \mathcal{F}}$, then

$$
\kappa_{1}-\kappa=d_{0,-1}^{*} \gamma
$$

for some $\gamma$ in $A_{1,1}$. Conversely, for any $\gamma$ in $A_{1,1}$, there exists a bundle-like metric $g_{1}$ on $(M, \mathcal{F})$ such that $\left.g_{1}\right|_{T \mathcal{F} \otimes T \mathcal{F}}=\left.g\right|_{T \mathcal{F} \otimes T \mathcal{F}}$ and (16) is satisfied. Moreover, if $\gamma$ is $C^{\infty}$-close to 0 , then we can take such $g_{1}$ so that $g_{1}$ is $C^{\infty}$-close to $g$.

The last sentence of Proposition 4.5 follows from the proof of Proposition 4.3 of Álvarez López 3 .

Let $(M, \mathcal{F})$ be a geometrically taut Riemannian flow. Let $g$ be a bundle-like metric on $M$. Let $*_{\mathcal{F}}: A_{0,1} \rightarrow A_{0,0}$ and $*_{\mathcal{F}}: A_{0,0} \rightarrow A_{0,1}$ be the leafwise Hodge

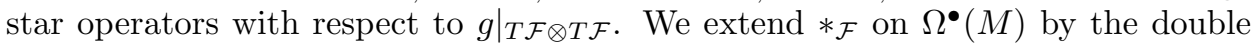
grading of $\Omega^{\bullet}(M)$ so that $*_{\mathcal{F}}$ acts trivially on $Q^{*}$. Thus $*_{\mathcal{F}}\left(A_{k, 1}\right)=A_{k, 0}$ and $*_{\mathcal{F}}\left(A_{k, 0}\right)=A_{k, 1}$. We show

Lemma 4.6. The restriction of $d_{0,-1}^{*}: A_{1,1} \rightarrow A_{1,0}$ to $\overline{d_{0,1}\left(A_{1,0}\right)}$ is a bijection $\overline{d_{0,1}\left(A_{1,0}\right)} \rightarrow \overline{d_{0,-1}^{*}\left(A_{1,1}\right)}$.

Proof. By [3, Theorem 2.1], we get

$$
A_{1,0}=\Omega_{b}^{1}(M / \mathcal{F}) \oplus \overline{d_{0,-1}^{*}\left(A_{1,1}\right)} .
$$

By operating $*_{\mathcal{F}}$ to both sides of (17), we get

$$
A_{1,1}=\left(\Omega_{b}^{1}(M / \mathcal{F}) \wedge \eta\right) \oplus \overline{d_{0,1}\left(A_{1,0}\right)} .
$$

The definition of basic forms implies that the kernel of $d_{0,1}: A_{1,0} \rightarrow A_{1,1}$ is the space $\Omega_{b}^{1}(M / \mathcal{F})$ of basic 1 -forms on $(M, \mathcal{F})$. By [3, Eq. 1.13], we get $d_{0,-1}^{*}=-*_{\mathcal{F}} d_{0,1} *_{\mathcal{F}}$ on $A_{1,1}$. Thus

$$
\operatorname{ker} d_{0,-1}^{*}=\Omega_{b}^{1}(M / \mathcal{F}) \wedge \eta \text {. }
$$

The proof is concluded by (17), (18) and (19). 
We will use the leafwise cohomology $H^{\bullet}(\mathcal{F})$ in the proof of the following lemma (see Section 3.4). As in the proof of Lemma 3.16, we will regard the $(0,1)$-th $E_{2}$-term $E_{2}^{0,1}(\mathcal{F})$ as a subspace of $H^{1}(\mathcal{F})$.

Proposition 4.7. If $\kappa$ is $C^{\infty}$-close to 0 , then there exists a Killing metric $g_{1}$ on $(M, \mathcal{F})$ which is $C^{\infty}$-close to $g$.

Proof. First we show that there exists a bundle-like metric $g_{2}$ on $(M, \mathcal{F})$ with characteristic form $\eta_{2}$ such that $g_{2}$ is $C^{\infty}$-close to $g$ and $\left.\eta_{2}\right|_{T \mathcal{F}}$ is the restriction of the characteristic form of a minimal bundle-like metric on $(M, \mathcal{F})$. By definition, there exists a minimal metric $g_{3}$ on $(M, \mathcal{F})$. Let $\eta_{3}$ be the characteristic form of $\left(M, \mathcal{F}, g_{3}\right)$. We will consider the leafwise cohomology classes $\left[\left.\eta\right|_{T \mathcal{F}}\right]$ and $\left[\left.\eta_{3}\right|_{T \mathcal{F}}\right]$ in $H^{1}(\mathcal{F})$. Here $E_{2}^{0,1}(\mathcal{F})$ is generated by $\left[\left.\eta_{3}\right|_{T \mathcal{F}}\right]$ by [30, Corollary 4.7]. Let $\overline{0}$ be the closure of 0 in $H^{1}(\mathcal{F})$, which may be nontrivial because $H^{1}(\mathcal{F})$ may be non-Hausdorff. Since $(M, \mathcal{F})$ is geometrically taut, [2, Proposition 3] implies that $E_{2}^{1,0}(\mathcal{F}) \rightarrow H^{1}(\mathcal{F}) / \overline{0}$ is surjective. Thus there exists $\varsigma$ in $C^{\infty}\left(T^{*} \mathcal{F}\right)$ such that $\varsigma$ is $C^{\infty}$-close to 0 and $\left[\left.\eta\right|_{T \mathcal{F}}\right]+[\varsigma]=\left[\left.\eta_{3}\right|_{T \mathcal{F}}\right]$. Here $\left.\eta\right|_{T \mathcal{F}}+\varsigma$ is nonzero at every point on $M$, because $\varsigma$ is $C^{\infty}$-close to 0 . Thus we get a smooth function $f$ on $M$ such that $\left.f \eta\right|_{T \mathcal{F}}=\left.\eta\right|_{T \mathcal{F}}+\varsigma$. Here $f$ is $C^{\infty}$-close to 1 , because $\varsigma$ is $C^{\infty}$-close to 0. Since $\left[\left.\eta_{3}\right|_{T \mathcal{F}}\right]$ belongs to $E_{2}^{0,1}(\mathcal{F})$, we get that $\left[\left.f \eta\right|_{T \mathcal{F}}\right]$ belongs to $E_{2}^{0,1}(\mathcal{F})$. Thus RummlerSullivan's criterion implies that $\left.f \eta\right|_{T \mathcal{F}}$ is the restriction of the characteristic form of a minimal bundle-like metric (see Sullivan [44, Rummler 42 and the Theorem in the introduction of Haefliger [26]). We take a bundle-like metric $g_{2}$ on $(M, \mathcal{F})$ so that the characteristic form is $f \eta$ and $\left.g_{2}\right|_{\operatorname{ker} \eta \otimes \operatorname{ker} \eta}=\left.g\right|_{\text {ker } \eta \otimes \operatorname{ker} \eta}$. Then $g_{2}$ is $C^{\infty}$ close to $g$ and the characteristic form $\left.f \eta\right|_{T \mathcal{F}}$ is the restriction of the characteristic form of a minimal bundle-like metric on $(M, \mathcal{F})$.

By the last paragraph, it is sufficient to show Proposition 4.7 in the case where $\left.\eta\right|_{T \mathcal{F}}$ is the restriction of the characteristic form of a minimal metric. Proposition 4.5 implies that there exists $\gamma$ in $A_{1,1}$ such that $\kappa=d_{0,-1}^{*} \gamma$. By Lemma 4.6 and the open mapping theorem, there exists a continuous inverse $\mu$ of $\left.d_{0,-1}^{*}\right|_{\overline{d_{0,1}\left(A_{1,0}\right)}}$ : $\overline{d_{0,1}\left(A_{1,0}\right)} \rightarrow \overline{d_{0,-1}^{*}\left(A_{1,1}\right)}$. Letting $\gamma=\mu(\kappa)$, we get $\gamma$ in $A_{1,1}$ such that $\kappa$ is $C^{\infty}$ close to 0 and $\kappa=d_{0,-1}^{*} \gamma$. Proposition 4.5 implies that we can take a minimal bundle-like metric $g_{1}$ which is $C^{\infty}$-close to $g$. Thus we get a minimal bundle-like metric $g_{1}$ which is $C^{\infty}$-close to $g$. Lemma 3.3 implies that $g_{1}$ is Killing.

4.3. Proof of Theorem 1.1. We recall a result in [38], which is essential in the following proof of Theorem 1.1

Theorem 4.8. Let $V$ be a connected open set of $\mathbb{R}^{\ell}$ and $\left\{\mathcal{F}^{t}\right\}_{t \in V}$ be a smooth family of Riemannian flows on a closed manifold. Then one of the following two cases occurs:

(i) $\mathcal{F}^{t}$ is geometrically taut for every $t$ in $V$.

(ii) $\mathcal{F}^{t}$ is not geometrically taut for every $t$ in $V$.

Proof of Theorem 1.1. We extend the smooth family of transverse metrics $\left\{g_{\nu}^{t}\right\}_{t \in V}$ to a smooth family of Riemannian metrics $\left\{\widehat{g}^{t}\right\}_{t \in V}$ on $M$ so that $\widehat{g}^{t}$ is bundle-like with respect to $\mathcal{F}^{t}$ and $\widehat{g}^{0}=g$. Here $\left(M, \mathcal{F}^{t}\right)$ is geometrically taut by Theorem 4.8 . Let $V_{1}$ be a sufficiently small open neighborhood of 0 in $V$. By Proposition 4.7. for $t$ in $V_{1}$, there exists a minimal metric $\widehat{g}_{1}^{t}$ on $\left(M, \mathcal{F}^{t}\right)$ for $t$ in $V_{1}$ such that $\widehat{g}_{1}^{t}$ is sufficiently close to $\widehat{g}^{t}$. 
We denote the formal adjoint operator of $d$ on $\left(M, \widehat{g}^{t}\right)$ by $d^{* t}$. We will show that there exists a 1 -form $\zeta^{t}$ such that

(I) $\zeta^{t}$ is sufficiently close to $\eta^{0}$,

(II) $d^{* t} \zeta^{t}=0$ and

(III) $d \zeta^{t}$ is a basic $(1,1)$-form on $\left(M, \mathcal{F}^{t}\right)$.

Let $\tau^{t}$ be the characteristic form of $\left(M, \mathcal{F}^{t}, \widehat{g}_{1}^{t}\right)$, which is close to $\eta^{0}$. Here $d \tau^{t}$ represents the basic Euler class of $\left(M, \mathcal{F}^{t}\right)$ by the definition. Since the $(0,2)$ component of the basic Euler class is trivial by the assumption, there exists a basic $(0,1)$-form $\sigma^{t}$ such that $\bar{\partial} \sigma^{t}=\left(d \tau^{t}\right)^{0,2}$. We consider $\sigma^{t}+\overline{\sigma^{t}}$ as a real 1-form on $M$. Let $\theta^{t}=\tau^{t}-\left(\sigma^{t}+\overline{\sigma^{t}}\right)$. Then $d \theta^{t}$ is a basic $(1,1)$-form. We consider a decomposition $\theta^{t}=\theta_{1}^{t}+\theta_{2}^{t}$, where $\theta_{1}^{t}$ is an exact 1 -form and $\theta_{2}$ belongs to ker $d^{* t}$ by the Hodge decomposition of $\Omega^{1}(M)_{\mathbb{C}}$ with respect to $\widehat{g}^{t}$. Let $\zeta^{t}=\theta_{2}^{t}$. It follows that $\theta_{1}^{t}$ is close to 0 , because $\tau^{t}$ is close to $\eta^{0}$, which belongs to ker $d^{* 0}$. Then condition (I) is satisfied. Condition (II) is clearly satisfied by the construction. Since $d \zeta^{t}=d \theta^{t}$ is a basic (1,1)-form, condition (III) is satisfied.

We consider differential operators $d_{1,1,-1}^{t}, D^{t}$ and $\Delta_{D}^{t}$ on $\left(M, \mathcal{F}^{t}\right)$ as in (4), (5), (6) and (7) using a splitting $T M=\left(H^{t}\right)^{1,0} \oplus\left(H^{t}\right)^{0,1} \oplus\left(T \mathcal{F}^{t} \otimes \mathbb{C}\right)$ determined by $H^{t}$ and $J^{t}$. Let $\mathbf{H}_{D}^{1}(t)$ be the space of $\Delta_{D}^{t}$-harmonic 1-forms. Let $\mathbf{H}^{1}(t)$ be the space of harmonic 1-forms on $\left(M, \widehat{g}^{t}\right)$. We show that $\operatorname{dim} \mathbf{H}_{D}^{1}(t)$ is constant with respect to $t$ on $V_{1}$. By Lemma 4.2, we have

$$
\operatorname{dim} \mathbf{H}_{D}^{1}(0)=\operatorname{dim} H^{1}(M ; \mathbb{C})+1 .
$$

Here $D^{t}$ is a self-adjoint strongly elliptic operator by Lemma 4.1 (i). Hence Theorem 4 of Kodaira and Spencer [33] implies that $\operatorname{dim} \mathbf{H}_{D}^{1}(t)$ is upper semicontinuous with respect to $t$. Thus we have

$$
\operatorname{dim} \mathbf{H}_{D}^{1}(t) \leq \operatorname{dim} \mathbf{H}_{D}^{1}(0)
$$

for $t$ in $V_{1}$. Here $\mathbf{H}^{1}(t) \subseteq \mathbf{H}_{D}^{1}(t)$ by Lemma 4.1(iv). By the previous paragraph, we have a 1-form $\zeta^{t}$ which satisfies conditions (I), (II) and (III) above. Condition (II) implies $D^{* t} \zeta^{t}=0$. Condition (III) implies $D^{t} \zeta^{t}=0$. Note that $\zeta^{t}$ is not $d$-closed by condition (I) and the nontriviality of $d \eta^{0}$. It follows that $\zeta^{t}$ is not contained in $\mathbf{H}^{1}(t)$. Thus we have

$$
\operatorname{dim} H^{1}(M ; \mathbb{C})+1 \leq \operatorname{dim} \mathbf{H}_{D}^{1}(t) .
$$

By (20), (21) and (22), we have

$$
\operatorname{dim} H^{1}(M ; \mathbb{C})+1=\operatorname{dim} \mathbf{H}_{D}^{1}(t) .
$$

Thus $\operatorname{dim} \mathbf{H}_{D}^{1}(t)$ is constant with respect to $t$.

Since $\operatorname{dim} \mathbf{H}_{D}^{1}(t)$ is constant with respect to $t$ by the consequence of the previous paragraph, the projection $F^{t}: \Omega^{1}(M)_{\mathbb{C}} \rightarrow \mathbf{H}_{D}^{1}(t)$ maps a smooth family of 1-forms to a smooth family of 1 -forms by [33, Theorem 5]. Letting $\varpi^{t}=F^{t}\left(\eta^{t}\right)$, we have a smooth family $\left\{\varpi^{t}\right\}_{t \in V_{1}}$ of $\Delta_{D}^{t}$-harmonic 1-forms such that $\varpi^{0}=F^{0}\left(\eta^{0}\right)=\eta^{0}$. We show that $d \varpi^{t}$ is a basic $(1,1)$-form on $\left(M, \mathcal{F}^{t}\right)$. By $D^{t} \varpi^{t}=0$, we have $d \varpi^{t}=$ $d_{1,1,-1}^{t} \varpi^{t}$. Thus $\iota_{\xi^{t}} d \varpi^{t}=0$. It follows that $d \varpi^{t}$ is basic. Hence $d \varpi^{t}$ is a basic $(1,1)-$ form on $\left(M, \mathcal{F}^{t}\right)$. Letting $\operatorname{Re} \varpi^{t}=\frac{\varpi^{t}+\bar{\varpi}^{t}}{2}$, we have a smooth family $\operatorname{Re} \varpi^{t}$ of real 1 -forms such that $d \operatorname{Re} \varpi^{t}$ is a basic $(1,1)$-form. Since $d \eta^{0}$ is nondegenerate on $T M / T \mathcal{F}^{0}, d \operatorname{Re} \varpi^{t}$ is also nondegenerate on $T M / T \mathcal{F}^{t}$ for $t$ in $V_{1}$. Thus $J^{t}$ and $d \operatorname{Re} \varpi^{t}$ determine a transverse metric on $T M / T \mathcal{F}^{t}$. By Proposition 2.16, a pair 
of a transversely Kähler flow $\left(\mathcal{F}^{t}, J^{t}\right)$ and a contact form Re $\varpi^{t}$ whose transverse Kähler form is $d \operatorname{Re} \varpi^{t}$ determines a Sasakian metric on $M$.

Remark 4.9. We remark that we can show the existence of a Sasakian metric on $M$ which is compatible with $\left(\mathcal{F}^{t}, J^{t}\right)$ for $t$ close to 0 directly by the triviality of the (0,2)-component of the basic Euler class without considering the Laplacian of $D$. But we need the operator $D$ to show the existence of a smooth family of compatible Sasakian metrics, which has a certain advantage compared with the existence of compatible Sasakian metrics at each parameter.

\section{Stability of K-COntact structures}

Let $M$ be a closed manifold with a Riemannian foliation $\mathcal{F}$ and a bundle-like metric $g$. In this section we consider only real differential forms. Let $Q=(T \mathcal{F})^{\perp}$. We use the well-known double grading $\Omega^{\bullet}(M)=\bigoplus_{j, k} A_{j, k}$ of the real de Rham complex $\Omega^{\bullet}(M)$, where $A_{j, k}=C^{\infty}\left(\bigwedge^{j} Q^{*} \otimes \bigwedge^{k} T^{*} \mathcal{F}\right)$ instead of the triple grading considered in Section 4.1. Then $d$ is decomposed in terms of the double grading as $d=d_{1,0}+d_{0,1}+d_{2,-1}$ as in (13). Let $\widehat{D}=d_{1,0}+d_{0,1}$. Since $d-\widehat{D}=d_{2,-1}$ is a differential operator of degree 0 by [3, Lemma 1.1], it follows that $\Delta_{\widehat{D}}$ is a self-adjoint strongly elliptic operator with the same symbol as the Laplacian of $d$. Let $\mathbf{H}_{\widehat{D}}^{1}$ be the space of $\Delta_{\widehat{D}}$-harmonic 1-forms. An argument similar to the proof of Lemma 4.2 shows

Lemma 5.1. If $(M, \mathcal{F})$ admits a $K$-contact structure whose Reeb flow is $\mathcal{F}$, then we have an equality

$$
\mathbf{H}_{\widehat{D}}^{1}=\mathbf{H}^{1} \oplus \mathbb{R} \eta
$$

in $\Omega^{1}(M)$.

Theorem 1.5 is proved by an argument similar to the proof of Theorem 1.1. We only describe the outline as follows:

Outline of the Proof of Theorem 1.5. Let $\left\{\widehat{g}^{t}\right\}_{t \in V}$ be a smooth family of Riemannian metrics on $M$ such that $\widehat{g}^{0}=g^{0}$ and the transverse metric of $\mathcal{F}^{t}$ induced from $\widehat{g}^{t}$ is $g_{\nu}^{t}$. Theorem 4.8 and Proposition 4.7 imply that there exists a Killing metric $\widehat{g}_{1}^{t}$ of $\left(M, \mathcal{F}^{t}\right)$ sufficiently close to $g^{t}$ for $t$ in a sufficiently small neighborhood $V_{1}$ of 0 in $V$.

Let $Q^{t}=\left(T \mathcal{F}^{t}\right)^{\perp}$. We consider a double grading $\Omega^{\bullet}(M)=\bigoplus_{j, k} A_{j, k}^{t}$ of $\Omega^{\bullet}(M)$, where $A_{j, k}^{t}=C^{\infty}\left(\bigwedge^{j} Q^{t *} \otimes \bigwedge^{k} T^{*} \mathcal{F}^{t}\right)$. We decompose $d$ by double grading on $\left(M, \mathcal{F}^{t}\right)$ and let $\widehat{D}^{t}=d_{1,0}^{t}+d_{0,1}^{t}$. As in the second paragraph of the proof of Theorem 1.1. we can show that there exists a 1 -form $\zeta^{t}$ on $M$ such that

(I') $\zeta^{t}$ is sufficiently close to $\eta^{0}$ and

(II') $d^{* t} \zeta^{t}=0$,

where $d^{* t}$ is the formal adjoint of $d$ on $\left(M, \widehat{g}^{t}\right)$.

We see that $\zeta^{t}$ is a $\widehat{D}^{t}$-harmonic form which is linearly independent of harmonic 1forms by an argument similar to the third paragraph of the proof of Theorem 1.1. Lemma 5.1 implies $\operatorname{dim} \mathbf{H}_{D}^{1}(t)=\operatorname{dim} \mathbf{H}^{1}(M ; \mathbb{R})+1$, where $\mathbf{H}_{D}^{1}(t)$ is the space of $\Delta_{\widehat{D}^{t}}$-harmonic 1-forms.

Since $\operatorname{dim} \mathbf{H}_{D}^{1}(t)$ is constant with respect to $t$, by [33, Theorem 5], we get a smooth family of 1 -forms $\varpi^{t}$ by projecting $\eta^{t}$ to $\mathbf{H}_{D}^{1}(t)$. Then $d \varpi^{t}$ is basic. Since 
$\varpi^{0}=\eta^{0}$ is a contact form, $\varpi^{t}$ is contact for $t$ in $V_{1}$. Thus $\varpi^{t}$ is a contact form whose Reeb flow is a Riemannian flow $\mathcal{F}^{t}$.

\section{KodAira-AKIZUKi-NAKANO VANishing THEOREM FOR TRANSVERSELY KÄHLER FOLIATIONS}

6.1. Basic Hodge star operator and basic Lefschetz operator. We recall a fundamental notion of Lefschetz theory for basic cohomology of transversely Kähler foliation introduced by El Kacimi Alaoui [19. Let $\left(\mathcal{F}, J, g_{\nu}\right)$ be a complex codimension $n$ transversely Kähler foliation with transverse Kähler form $\omega$. We consider complex differential forms $\Omega^{\bullet}(M)_{\mathbb{C}}=\Omega^{\bullet}(M) \otimes \mathbb{C}$ throughout Section 6. At each point $x$ on $M$, we have the Hodge star operator

$$
*_{b, x}: \bigwedge^{k}\left(T_{x}^{*} M / T_{x}^{*} \mathcal{F}\right) \otimes \mathbb{C} \longrightarrow \bigwedge^{2 n-k}\left(T_{x}^{*} M / T_{x}^{*} \mathcal{F}\right) \otimes \mathbb{C}
$$

determined by the transverse metric $g$ on $T_{x} M / T_{x} \mathcal{F}$. A basic $k$-form on $(M, \mathcal{F})$ can be regarded as a section of $\bigwedge^{k}\left(T^{*} M / T^{*} \mathcal{F}\right)$. Thus we have the basic Hodge operator

$$
*_{b}: \Omega_{b}^{k}(M / \mathcal{F})_{\mathbb{C}} \longrightarrow \Omega_{b}^{2 n-k}(M / \mathcal{F})_{\mathbb{C}}
$$

Composing $*_{b}$ with complex conjugation, we have

$$
\bar{\star}_{b}: \Omega_{b}^{p, q}(M / \mathcal{F}) \longrightarrow \Omega_{b}^{n-p, n-q}(M / \mathcal{F}) .
$$

Let $\left(E, h_{E}\right)$ be a holomorphic $\mathcal{F}$-fibered Hermitian line bundle over $(M, \mathcal{F})$. Let

$$
h: E \longrightarrow E^{*}
$$

be a $\mathbb{C}$-antilinear isomorphism defined by $h(s)=h_{E}(s, \cdot)$. The complex conjugation of the basic Hodge star operator on the basic Dolbeault complex with values in $E$,

$$
\bar{*}_{b, E}: \Omega_{b}^{p, q}(M / \mathcal{F}, E) \longrightarrow \Omega_{b}^{n-p, n-q}\left(M / \mathcal{F}, E^{*}\right),
$$

is defined by $\bar{*}_{b, E}(\alpha \otimes s)=\bar{*}_{b} \alpha \otimes h(s)$ for sections of the form $\alpha \otimes s$, where $\alpha$ is a basic $(p, q)$-form and $s$ is a local holomorphic section of $E$. We define the basic Lefschetz operator

$$
L: \Omega_{b}^{k}(M / \mathcal{F}, E)_{\mathbb{C}} \longrightarrow \Omega_{b}^{k+2}(M / \mathcal{F}, E)_{\mathbb{C}}
$$

by $L \alpha=\alpha \wedge \omega$, where $\omega$ is the transverse Kähler form of $\left(M, \mathcal{F}, J, g_{\nu}\right)$.

6.2. Chern connections of $\mathcal{F}$-fibered Hermitian holomorphic line bundles. Let $\left(E, h_{E}\right)$ be a holomorphic $\mathcal{F}$-fibered Hermitian line bundle over $(M, \mathcal{F})$. Let

$$
\nabla_{E}: \Omega_{b}^{k}(M / \mathcal{F}, E)_{\mathbb{C}} \longrightarrow \Omega_{b}^{k+1}(M / \mathcal{F}, E)_{\mathbb{C}}
$$

be a basic connection of $\left(E, h_{E}\right)$. Recall that

$$
\nabla_{E}\left(\Omega_{b}^{p, q}(M / \mathcal{F}, E)\right) \subseteq \Omega_{b}^{p+1, q}(M / \mathcal{F}, E) \oplus \Omega_{b}^{p, q+1}(M / \mathcal{F}, E) .
$$

We define the $(1,0)$-component $\nabla_{E}^{\prime}$ and the $(0,1)$-component $\nabla_{E}^{\prime \prime}$ of $\nabla_{E}$ by

$$
\nabla_{E}^{\prime} \alpha=\pi^{p+1, q} \nabla_{E} \alpha, \quad \nabla_{E}^{\prime \prime} \alpha=\pi^{p, q+1} \nabla_{E} \alpha
$$

for $\alpha$ in $\Omega_{b}^{p, q}(M / \mathcal{F}, E)$, where $\pi^{i, j}$ is the projection to $\Omega_{b}^{i, j}(M / \mathcal{F}, E)$ for $(i, j)=$ $(p+1, q)$ and $(p, q+1)$, respectively. Throughout Section 6 we assume that $\nabla_{E}$ is the Chern connection of $\left(E, h_{E}\right)$, that is, $\nabla_{E}$ is the unique connection on $E$ such that $\nabla_{E} h=0$ and $\nabla_{E}^{\prime \prime}=\bar{\partial}_{E}$, where $\bar{\partial}_{E}$ is the basic Dolbeault operator defined in Definition 3.9. In particular, $\left(\nabla_{E}^{\prime \prime}\right)^{2}=0$. 
Regarding the curvature form $\Theta\left(\nabla_{E}\right)$ of $\nabla_{E}$ as a basic 2-form on $(M, \mathcal{F})$ by a natural isomorphism

$$
\Omega_{b}^{2}(M / \mathcal{F})_{\mathbb{C}} \otimes \operatorname{End}(E) \cong \Omega_{b}^{2}(M / \mathcal{F})_{\mathbb{C}} \otimes C_{b}^{\infty}(M / \mathcal{F})_{\mathbb{C}} \cong \Omega_{b}^{2}(M / \mathcal{F})_{\mathbb{C}},
$$

we define the curvature operator

$$
\Theta\left(\nabla_{E}\right): \Omega_{b}^{k}(M / \mathcal{F}, E)_{\mathbb{C}} \longrightarrow \Omega_{b}^{k+2}(M / \mathcal{F}, E)_{\mathbb{C}}
$$

of $\nabla_{E}$ by $\Theta\left(\nabla_{E}\right) \alpha=\alpha \wedge \Theta\left(\nabla_{E}\right)$.

Note that there exists a unique connection $\nabla_{E^{*}}$ on $E^{*}$ which satisfies

$$
d\left\langle s, s^{*}\right\rangle=\left\langle\nabla_{E} s, s^{*}\right\rangle+\left\langle s, \nabla_{E^{*}} s^{*}\right\rangle,
$$

for $s$ in $C^{\infty}(E)$ and $s^{*}$ in $C^{\infty}\left(E^{*}\right)$ where $\langle\cdot, \cdot\rangle$ is the coupling $E \otimes E^{*} \rightarrow \mathbb{C}$.

Let $\widetilde{\wedge}$ be the wedge product defined by the composite of

$$
\Omega_{b}^{p, q}(M / \mathcal{F}, E) \times \Omega_{b}^{n-p, n-q}\left(M / \mathcal{F}, E^{*}\right) \stackrel{\wedge}{\longrightarrow} \Omega_{b}^{n, n}\left(M / \mathcal{F}, E \otimes E^{*}\right) \longrightarrow \Omega_{b}^{n, n}(M / \mathcal{F}),
$$

where the second map is induced by the coupling $E \otimes E^{*} \rightarrow \mathbb{C}$.

6.3. Homological orientability of transversely Kähler foliations. Here we recall a terminology due to El Kacimi Alaoui [19]. Let $\left(\mathcal{F}, J, g_{\nu}\right)$ be a transversely Kähler foliation of complex codimension $n$ on a closed manifold $M$.

Definition 6.1. $\left(\mathcal{F}, J, g_{\nu}\right)$ is homologically orientable if the basic cohomology group $H_{b}^{2 n}(M / \mathcal{F})$ of degree $2 n$ is nontrivial.

We refer to Masa 34 or El Kacimi Alaoui and Hector 20 for the basic cohomology of Riemannian foliations. Since the basic cohomology is determined only by the underlying foliation $\mathcal{F}$, it is independent of the transverse structures. By a theorem of Masa [34] in the general case, or by a theorem of Molino and Sergiescu [37] in the case of flows, $\left(\mathcal{F}, g_{\nu}\right)$ is homologically orientable if and only if $\mathcal{F}$ is geometrically taut (see Definition 3.2).

Example 6.2. The Reeb flow $\mathcal{F}$ of a Sasakian manifold is isometric (see Definition 3.1). Then it is geometrically taut by Lemma 3.3 due to Carrière. Thus $\mathcal{F}$ is homologically orientable by a theorem of Masa or a theorem of Molino and Sergiescu.

6.4. An inner product of El Kacimi Alaoui-Hector. We assume that $(M, \mathcal{F})$ is homologically orientable in the sequel (see Definition 6.1). We will use the Molino theory, which is one of the fundamental tools in the work of El Kacimi Alaoui and Hector [20] and El Kacimi Alaoui [19]. We refer to Molino [36] for the Molino theory. We define an inner product on $\Omega_{b}^{\bullet}(M / \mathcal{F}, E)_{\mathbb{C}}$ under the assumption of homological orientability following the argument of El Kacimi Alaoui and Hector in 20, Section 4.5] (see also El Kacimi Alaoui [19]), where they consider the case of trivial line bundles Let $\rho: M^{1} \rightarrow M$ be the orthonormal frame bundle of the normal bundle of $\mathcal{F}$. Let $\pi: M^{1} \rightarrow W$ be the basic fibration of $\mathcal{F}$. Let $m=\operatorname{dim} \operatorname{SO}(2 n)$ and $X_{1}$, $X_{2}, \ldots, X_{m}$ be the vector fields on $M$ which generate the principal $\mathrm{SO}(2 n)$-action on $M^{1}$. Let $\theta_{i}$ be the basic 1 -form on $\left(M^{1}, \mathcal{F}^{1}\right)$ dual to $X_{i}$. We define an $m$-form $\chi$ on $M^{1}$ by

$$
\chi=\theta_{1} \wedge \theta_{2} \wedge \cdots \wedge \theta_{m}
$$


If $W$ is orientable, an inner product $(\cdot, \cdot)$ is defined by

$$
\left(\alpha_{1}, \alpha_{2}\right)=\int_{W} \mathcal{I}\left(\rho^{*}\left(\alpha_{1} \widetilde{\wedge}^{*} b, E \alpha_{2}\right) \wedge \chi\right)
$$

for $\alpha_{1}$ and $\alpha_{2}$ in $\Omega_{b}^{\bullet}(M / \mathcal{F}, E)_{\mathbb{C}}$. Here $\mathcal{I}: \Omega_{b}^{k}(M / \mathcal{F})_{\mathbb{C}} \rightarrow \Omega^{k-d}(W)_{\mathbb{C}}$ is the integration along fibers of $\pi$ defined under the assumption of the homological orientability of $\mathcal{F}$ by El Kacimi Alaoui and Hector (see [20, Proposition 3.2]). This $\mathcal{I}$ commutes with $d$ as shown there. If $W$ is not orientable, the orientation cover of $W$ can be used to define an inner product in the same equation as (25). In what follows, we also assume the orientability of $W$ for simplicity.

Lemma 6.3. We have

$$
\left(\nabla_{E} \alpha_{1}, \alpha_{2}\right)=\left(\alpha_{1},-\left(\bar{*}_{b, E^{*}}\right) \nabla_{E^{*}}\left(\bar{*}_{b, E}\right) \alpha_{2}\right)
$$

for $\alpha_{1}$ in $\Omega_{b}^{k-1}(M / \mathcal{F}, E)_{\mathbb{C}}$ and $\alpha_{2}$ in $\Omega_{b}^{k}(M / \mathcal{F}, E)_{\mathbb{C}}$, where $\nabla_{E^{*}}$ is the connection dual to $\nabla_{E}$ mentioned in (23).

Proof. We closely follow the argument in [20, Proposition 4.6] or [19, Section 3.2.4], where they consider the case of trivial bundles. Letting $\beta_{1}=\bar{*}_{b, E} \alpha_{2}$, we have

$$
\begin{aligned}
& \text { (27) } d\left(\rho^{*}\left(\alpha_{1} \widetilde{\wedge} \beta_{1}\right) \wedge \chi\right) \\
& =\left(\rho^{*}\left(\nabla_{E} \alpha_{1} \widetilde{\wedge} \beta_{1}\right) \wedge \chi\right)+(-1)^{k-1}\left(\rho^{*}\left(\alpha_{1} \widetilde{\wedge} \nabla_{E^{*}} \beta_{1}\right) \wedge \chi\right)+(-1)^{2 n-1}\left(\rho^{*}\left(\alpha_{1} \widetilde{\wedge} \beta_{1}\right) \wedge d \chi\right),
\end{aligned}
$$

where $2 n$ is the real codimension of $\mathcal{F}$. Recall that $\rho: M^{1} \rightarrow M$ is the projection. Let $A_{j, k}\left(M^{1}\right)=C^{\infty}\left(\bigwedge^{j}(\operatorname{ker} \chi)^{*} \otimes \bigwedge^{k}(\operatorname{ker} T \rho)^{*}\right)$, where $T \rho$ is the differential map of $\rho$. Then we get a double grading $\Omega^{\bullet}\left(M^{1}\right)_{\mathbb{C}}=\bigoplus_{j, k} A_{j, k}\left(M^{1}\right)$. Here $d$ is decomposed into $d=d_{0,1}+d_{1,0}+d_{2,-1}$, where the subscripts means the grading. Recall that we let $m=\operatorname{dim} \mathrm{SO}(2 n)$. Since $\chi$ is of degree $(0, m)$, we can decompose $d \chi$ as

$$
d \chi=d_{0,1} \chi+d_{1,0} \chi+d_{2,-1} \chi
$$

where $d_{i, j} \chi$ belongs to $A_{i, j+m}\left(M^{1}\right)$. We get $d_{0,1} \chi=0$, because $A_{\bullet, m+1}\left(M^{1}\right)=0$. By Rummler's formula (see the second formula in the proof of [42, Proposition 1] or [11, Lemma 10.5.6]), we get $d_{1,0} \chi=-\chi \wedge \kappa_{\rho}$, where $\kappa_{\rho}$ is the mean curvature form of the foliation defined by the fibers of $\rho$. By the definition of $\chi$, we get $\kappa_{\rho}=0$, which implies $d_{1,0} \chi=0$. Thus $d \chi$ is of degree $(2, m-1)$. Since $A_{2 n+1, \bullet}\left(M^{1}\right)=0$, we get $d \chi \wedge \alpha=0$ for any $\alpha$ in $A_{2 n-1,0}\left(M^{1}\right)$, which implies that the third term of the right hand side of (27) is zero. Composing $\mathcal{I}$ and $\int_{W}$ to both sides of (27), we have (26).

6.5. Formal adjoint operators with respect to $(\cdot, \cdot)$. Recall that the formal adjoint $\mathcal{D}^{\star}$ of a $\mathbb{C}$-linear map $\mathcal{D}: \Omega_{b}^{\bullet}(M / \mathcal{F}, E)_{\mathbb{C}} \rightarrow \Omega_{b}^{\bullet+k}(M / \mathcal{F}, E)_{\mathbb{C}}$ is a $\mathbb{C}$-linear map $\mathcal{D}^{\star}: \Omega_{b}^{\bullet}(M / \mathcal{F}, E)_{\mathbb{C}} \rightarrow \Omega_{b}^{\bullet-k}(M / \mathcal{F}, E)_{\mathbb{C}}$ such that $\left(\mathcal{D} \alpha_{1}, \alpha_{2}\right)=\left(\alpha_{1}, \mathcal{D}^{\star} \alpha_{2}\right)$ for $\alpha_{1}$ in $\Omega_{b}^{\bullet}(M / \mathcal{F}, E)_{\mathbb{C}}$ and $\alpha_{2}$ in $\Omega_{b}^{\bullet+k}(M / \mathcal{F}, E)_{\mathbb{C}}$. The following lemma shows that the formal adjoint operators with respect to $(\cdot, \cdot)$ are given by conjugation by the Hodge star operators as in the case of complex manifolds. This is an advantage of the inner product $(\cdot, \cdot)$, which allows us to apply the classical argument for complex manifolds to show vanishing theorems: 
Lemma 6.4. Letting $\Lambda=L^{\star}$, we get

$$
\begin{aligned}
\Lambda & =\left(\bar{*}_{b, E^{*}}\right) L\left(\bar{*}_{b, E}\right), \\
\nabla_{E}^{\prime \prime \star} & =-\left(\bar{*}_{b, E^{*}}\right) \nabla_{E^{*}}^{\prime \prime}\left(\bar{*}_{b, E}\right), \\
\nabla_{E}^{\prime \star} & =-\left(\bar{*}_{b, E^{*}}\right) \nabla_{E^{*}}^{\prime}\left(\bar{*}_{b, E}\right) .
\end{aligned}
$$

Proof. For $\alpha_{1}$ in $\Omega_{b}^{k}(M / \mathcal{F}, E)_{\mathbb{C}}$ and $\alpha_{2}$ in $\Omega_{b}^{k+2}(M / \mathcal{F}, E)_{\mathbb{C}}$, we get

$\left(\alpha_{1}, \Lambda \alpha_{2}\right)=\left(L \alpha_{1}, \alpha_{2}\right)=\left(\alpha_{1} \wedge \omega, \alpha_{2}\right)=\left(\alpha_{1}, \bar{*}_{b, E^{*}}\left(\omega \wedge \bar{*}_{b, E} \alpha_{2}\right)\right)=\left(\alpha_{1}, \bar{*}_{b, E^{*}} L \bar{*}_{b, E} \alpha_{2}\right)$

by the definition of $(\cdot, \cdot)$. Then (28) is proved. Take $\alpha_{3}$ in $\Omega_{b}^{p, q-1}(M / \mathcal{F}, E)$ and $\alpha_{4}$ in $\Omega_{b}^{p, q}(M / \mathcal{F}, E)$. We get $\left(\nabla_{E}^{\prime} \alpha_{3}, \alpha_{4}\right)=0$ and $\left(\alpha_{3}, \bar{*}_{b}, E^{*} \nabla_{E^{*}}^{\prime} \bar{*}_{b}, E \alpha_{4}\right)=0$ because of the degree. Thus Lemma 6.3 implies

$$
\left(\nabla_{E}^{\prime \prime} \alpha_{3}, \alpha_{4}\right)=\left(\nabla_{E} \alpha_{3}, \alpha_{4}\right)=\left(\alpha_{3},-\bar{*}_{b, E^{*}} \nabla_{E^{*} \bar{*}_{b, E}} \alpha_{4}\right)=\left(\alpha_{3},-\bar{*}_{b, E^{*}} \nabla_{E^{*} \bar{*}_{b}, \alpha_{4}}^{\prime \prime}\right) .
$$

Thus (29) is proved. Take $\alpha_{5}$ in $\Omega_{b}^{p-1, q}(M / \mathcal{F}, E)$ and $\alpha_{6}$ in $\Omega_{b}^{p, q}(M / \mathcal{F}, E)$. Because of the degree, we get $\left(\nabla_{E}^{\prime \prime} \alpha_{5}, \alpha_{6}\right)=0$ and $\left(\alpha_{5}, \bar{*}_{b, E^{*}} \nabla_{E^{*}}^{\prime \prime} \bar{*}_{b, E} \alpha_{6}\right)=0$. Thus Lemma 6.3 implies

$$
\left(\nabla_{E}^{\prime} \alpha_{5}, \alpha_{6}\right)=\left(\nabla_{E} \alpha_{5}, \alpha_{6}\right)=\left(\alpha_{5},-\bar{*}_{b, E^{*}} \nabla_{E^{*} \bar{*}_{b, E}} \alpha_{6}\right)=\left(\alpha_{5},-\bar{*}_{b, E^{*}} \nabla_{E^{*} \bar{*}_{b}, E} \alpha_{6}\right) .
$$

Thus (30) is proved.

Remark 6.5. $\Omega_{b}^{\bullet \bullet \bullet}(M / \mathcal{F}, E)$ has another natural inner product obtained by the restriction of the inner product on $\Omega^{\bullet}(M, E)_{\mathbb{C}}$. In this case, the formal adjoint of

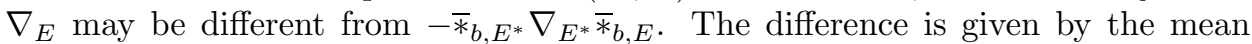
curvature form of $\mathcal{F}$ (see Proposition 3.6 of Kamber and Tondeur [30] for the case where $E$ is trivial).

6.6. Bochner-Kodaira-Nakano identity. For each integer $k$, let

$$
\mathfrak{g}_{k}=\left\{A \in \operatorname{End}_{\mathbb{C}-\operatorname{Vect}}\left(\Omega_{b}^{\bullet}(M / \mathcal{F}, E)_{\mathbb{C}}\right) \mid A\left(\Omega_{b}^{\bullet}(M / \mathcal{F}, E)_{\mathbb{C}}\right) \subseteq \Omega_{b}^{\bullet+k}(M / \mathcal{F}, E)_{\mathbb{C}}\right\} .
$$

It is well known that $\mathfrak{g}=\bigoplus_{k \in \mathbb{Z}} \mathfrak{g}_{k}$ has a bracket operation $[\cdot, \cdot]: \mathfrak{g} \times \mathfrak{g} \rightarrow \mathfrak{g}$ defined by $[A, B]=A B-(-1)^{a \cdot b} B A$ for $A$ in $\mathfrak{g}_{a}$ and $B$ in $\mathfrak{g}_{b}$. The Laplacian of $\nabla_{E}^{\prime \prime}$ and $\nabla_{E}^{\prime}$ is defined by

$$
\Delta_{E}^{\prime \prime}=\left[\nabla_{E}^{\prime \prime}, \nabla_{E}^{\prime \prime \star}\right], \quad \Delta_{E}^{\prime}=\left[\nabla_{E}^{\prime}, \nabla_{E}^{\prime \star}\right] .
$$

The Bochner-Kodaira-Nakano identity describes the difference of two Laplacians by the curvature of $E$. For the proof of the identity, we recall our notation on the local description of basic forms on $(M, \mathcal{F})$ in Section 3.2 Let $\left(M, \mathcal{F}, J, g_{\nu}\right)$ be a transversely Kähler foliation. Let $(E, h)$ be a holomorphic $\mathcal{F}$-fibered Hermitian line bundle on $(M, \mathcal{F})$. As in Section 3.2, we take a covering $\left\{U_{j}\right\}$ of $M$ so that $\left(U_{j},\left.\mathcal{F}\right|_{U_{j}}\right)$ is isomorphic to the standard transversely holomorphic foliation defined by a decomposition $\mathbb{R}^{m} \times B_{j}^{n}=\bigsqcup_{z \in B_{j}^{n}} \mathbb{R}^{m} \times\{z\}$, where $B_{j}^{n}$ is the unit ball of $\mathbb{C}^{n}$. Let $\phi_{j}: U_{j} \rightarrow B_{j}^{n}$ be the composite of $U_{j} \cong \mathbb{R}^{m} \times B_{j}^{n} \rightarrow B_{j}^{n}$, where the second map is the second projection. By definition of holomorphic $\mathcal{F}$-fibered Hermitian line bundle (see Definition 3.4), there exists a Hermitian holomorphic line bundle $\left(E_{j}, h_{j}\right)$ and a Kähler metric $g_{j}$ on $B_{j}^{n}$ which determines $g_{\nu}, E$ and $h$ by

$$
\begin{aligned}
\left.g_{\nu}\right|_{U_{j}} & =\phi_{j}^{*} g_{j}, \\
\left.E\right|_{U_{j}} & =\phi_{j}^{*} E_{j}, \\
\left.h\right|_{U_{j}} & =\phi_{j}^{*} h_{j} .
\end{aligned}
$$


We consider the Chern connection $\nabla_{j}$ of $\left(E_{j}, h_{j}\right)$, the Lefschetz operator $L_{j}$ on $B_{j}^{n}$ and the complex conjugate of the Hodge star operator $\bar{*}_{E_{j}}$ on $B_{j}^{n}$ with values in $E_{j}$. We denote the curvature operator of $\nabla_{j}$ by $\Theta\left(\nabla_{j}\right)$. Clearly we get

$$
\begin{aligned}
\left(\bar{*}_{b, E}\right) \phi_{j}^{*} \beta & =\phi_{j}^{*}\left(\bar{*}_{E_{j}} \beta\right), \\
L \phi_{j}^{*} \beta & =\phi_{j}^{*}\left(L_{j} \beta\right), \\
\nabla_{E}^{\prime} \phi_{j}^{*} \beta & =\phi_{j}^{*}\left(\nabla_{j}^{\prime} \beta\right), \\
\nabla_{E}^{\prime \prime} \phi_{j}^{*} \beta & =\phi_{j}^{*}\left(\nabla_{j}^{\prime \prime} \beta\right), \\
\Theta\left(\nabla_{E}\right) \phi_{j}^{*} \beta & =\phi_{j}^{*}\left(\Theta\left(\nabla_{j}\right) \beta\right)
\end{aligned}
$$

for a differential form $\beta$ on $B_{j}^{n}$ valued in $E_{j}$. We have the following BochnerKodaira-Nakano identity for homologically orientable transversely Kähler foliations:

Lemma 6.6. We get

$$
\Delta_{E}^{\prime \prime}=\Delta_{E}^{\prime}+\left[\sqrt{-1} \Theta\left(\nabla_{E}\right), \Lambda\right],
$$

where $\Delta_{E}^{\prime \prime}=\left[\nabla_{E}^{\prime \prime}, \nabla_{E}^{\prime \prime \star}\right]$ and $\Delta_{E}^{\prime}=\left[\nabla_{E}^{\prime}, \nabla_{E}^{\prime \star}\right]$.

Proof. We consider $U_{j}$ and $\phi_{j}: U_{j} \rightarrow B_{j}^{n}$ mentioned above in this subsection. Since the formula is local, we can consider each $U_{j}$. By the Bochner-Kodaira-Nakano identity (see Sections 4.5 and 4.6 of Demailly [15] or Theorem 1.1 of Chapter VII of Demailly [16]) for the Hermitian holomorphic line bundle $E_{j}$ on $B_{j}^{n}$, we have

$$
\Delta_{E_{j}}^{\prime \prime}=\Delta_{E_{j}}^{\prime}+\left[\sqrt{-1} \Theta\left(\nabla_{j}\right), \Lambda_{j}\right]
$$

where $\Lambda_{j}=\bar{*}_{E_{j}^{*}} L_{j} \bar{*}_{E_{j}}$. Note that (33) is proved only by local calculation on a complex coordinate chart, which can be applied to $E_{j}$ and $B_{j}^{n}$ in our situation. Then (32) is proved by pulling back (33) by $\phi_{j}$ by (28), (29), (30) of Lemma 6.4 and the five equations in (31).

6.7. Hodge decomposition for the basic Dolbeault complex valued on $E$. The Hodge decomposition theorem of El Kacimi Alaoui is as follows in this context:

Theorem 6.7 (El Kacimi Alaoui [19, Theorem 2.8.7]). There is an orthogonal decomposition

$$
\Omega_{b}^{p, q}(M / \mathcal{F}, E)=\operatorname{ker} \Delta_{E}^{\prime \prime} \oplus \operatorname{Im} \nabla_{E}^{\prime \prime} \oplus \operatorname{Im} \nabla_{E}^{\prime \prime \star}
$$

with respect to the inner product $(\cdot, \cdot)$, where $\Delta_{E}^{\prime \prime}=\left[\nabla_{E}^{\prime \prime}, \nabla_{E}^{\prime \prime}\right]$.

Outline of the proof of Theorem 6.7. Let

$$
E^{p, q}=\wedge^{p}\left(T^{*} M / T^{*} \mathcal{F}\right)^{1,0} \otimes \wedge^{q}\left(T^{*} M / T^{*} \mathcal{F}\right)^{0,1} \otimes E .
$$

Then $\Omega_{b}^{p, q}(M / \mathcal{F}, E)$ is identified with the space $C^{\infty}\left(E^{p, q}, \mathcal{F}\right)$ of global basic sections of $E^{p, q}$ on $(M, \mathcal{F})$. Let $\rho: M^{1} \rightarrow M$ be the orthonormal frame bundle of $T M / T \mathcal{F}$. We denote the horizontal lift of $\mathcal{F}$ to $M^{1}$ by $\mathcal{F}^{1}$. Let $M^{1} \rightarrow W$ be the basic fibration of $\mathcal{F}$. Let $E^{1}=\rho^{*} E^{p, q}$. Here $E^{1}$ is $\mathcal{F}^{1}$-fibered. $C^{\infty}\left(E^{p, q}, \mathcal{F}\right)$ is identified with the space $C^{\infty}\left(E^{1}, \mathcal{F}^{1}\right)^{\mathrm{SO}(2 n)}$ of the $\mathrm{SO}(2 n)$-invariant basic section of $E^{1}$. The restriction of a global basic section $s$ of $E^{1}$ to the closure $\bar{L}$ of a leaf of $\mathcal{F}^{1}$ is determined by $s(x)$ at a point $x$ in $\bar{L}$. Thus $C^{\infty}\left(E^{1}, \mathcal{F}^{1}\right)$ can be identified with the space $C_{W}^{\infty}(\bar{E})$ of global sections of a vector bundle $\bar{E}$ over $W$. The inner product $(\cdot, \cdot)$ induces a Hermitian metric of $\bar{E}$. El Kacimi Alaoui constructed a differential operator $\mathcal{D}^{\prime}: C^{\infty}\left(E^{1}, \mathcal{F}^{1}\right) \rightarrow C^{\infty}\left(E^{1}, \mathcal{F}^{1}\right)$, which is identified with a self-adjoint strongly elliptic operator $\overline{\mathcal{D}}: C_{W}^{\infty}(\bar{E}) \rightarrow C_{W}^{\infty}(\bar{E})$ and whose 
restriction to $C^{\infty}\left(E^{1}, \mathcal{F}^{1}\right)^{\mathrm{SO}(2 n)}$ is identified with $\Delta_{E}^{\prime \prime}$. Then the Hodge decomposition $C^{\infty}\left(E^{p, q}, \mathcal{F}\right)=\operatorname{ker} \Delta_{E}^{\prime \prime} \oplus \operatorname{Im} \Delta_{E}^{\prime \prime}$ for $\Delta_{E}^{\prime \prime}$ is obtained by the restriction of the classical Hodge decomposition $C^{\infty}(\bar{E})=\operatorname{ker} \overline{\mathcal{D}} \oplus \operatorname{Im} \overline{\mathcal{D}}$ for $\overline{\mathcal{D}}$ to $C^{\infty}(\bar{E})^{\mathrm{SO}(2 n)}$. The equality $\operatorname{Im} \Delta_{E}^{\prime \prime}=\operatorname{Im} \nabla_{E}^{\prime \prime} \oplus \operatorname{Im} \nabla_{E}^{\prime \prime *}$ in $\Omega_{b}^{p, q}(M / \mathcal{F}, E)$ follows from the decompositions $\Omega_{b}^{i, j}(M / \mathcal{F}, E)=\operatorname{ker} \Delta_{E}^{\prime \prime} \oplus \operatorname{Im} \Delta_{E}^{\prime \prime}$ for $(i, j)=(p, q-1),(p, q+1)$ and $\Delta_{E}^{\prime \prime}=\left(\nabla_{E}^{\prime \prime}+\nabla_{E}^{\prime \prime \star}\right)^{2}$.

6.8. Serre duality valued on $E$. By (26) in Lemma 6.3, the pairing $\widetilde{\wedge}$ defined in (24) induces a product on basic Dolbeault cohomology

$$
H_{b}^{p, q}(M / \mathcal{F}, E) \times H_{b}^{n-p, n-q}\left(M / \mathcal{F}, E^{*}\right) \longrightarrow \mathbb{C} .
$$

The Serre duality follows from the Hodge decomposition Theorem 6.7 and the formula of formal adjoint operators in Lemma 6.4 as in the case of complex manifolds:

Theorem 6.8. The pairing (34) is nondegenerate, which implies $H_{b}^{p, q}(M / \mathcal{F}, E) \cong$ $H_{b}^{n-p, n-q}\left(M / \mathcal{F}, E^{*}\right)^{*}$.

Proof. Let $\mathbf{H}_{b}^{p, q}(E)$ (resp. $\mathbf{H}_{b}^{p, q}\left(E^{*}\right)$ ) be the space of basic harmonic $(p, q)$-forms valued on $E$ (resp. $\left.E^{*}\right)$. For each $\alpha$ in $\mathbf{H}_{b}^{p, q}(E)$, we have $\int_{M} \mathcal{I}\left(\alpha \widetilde{\wedge}^{*}{ }_{b, E} \alpha \wedge \chi\right) \neq 0$. Equation (29) of Lemma 6.4 implies $\Delta_{E}^{\prime \prime}=\bar{*}_{b, E^{*}} \Delta_{E^{*}}^{\prime \prime} \bar{*}_{b, E}$. Thus $\bar{*}_{b, E} \alpha \in \mathbf{H}_{b}^{n-p, n-q}\left(E^{*}\right)$. Then the pairing $\mathbf{H}^{p, q}(E) \times \mathbf{H}^{n-p, n-q}\left(E^{*}\right) \rightarrow \mathbb{C}$ defined by the restriction of (34) is nondegenerate, which concludes the proof of Theorem 6.8.

6.9. $\mathcal{F}$-fibered positive line bundles. Let $\left(E, h_{E}\right)$ be a holomorphic $\mathcal{F}$-fibered Hermitian line bundle on $(M, \mathcal{F})$. We recall the definition of the positivity of $\left(E, h_{E}\right)$ due to Boyer, Galicki and Nakamaye [10, which is defined in a way similar to the case of line bundles on complex manifolds:

Definition 6.9. $E$ is called positive if there exists a Hermitian metric $h$ on $E$ such that the basic first Chern form $\frac{\sqrt{-1}}{2 \pi} \Theta\left(\nabla_{E}\right)$ is a transverse Kähler form of $(\mathcal{F}, J)$ for a transverse metric on $(M, \mathcal{F})$.

The positivity of $E$ is determined by the first Chern class as follows:

Proposition 6.10 ([19, Section 3.5.6]). If there exists a transverse Kähler metric on $T M / T \mathcal{F}$ with transverse Kähler form $\omega$ such that $\left[\frac{\sqrt{-1}}{2 \pi} \Theta\left(\nabla_{E}\right)\right]=[\omega]$ in $H_{b}^{2}(M / \mathcal{F})$, then there exists a Hermitian metric $h^{\prime}$ on $E$ such that $(E, \widehat{h})$ is a holomorphic $\mathcal{F}$-fibered Hermitian line bundle whose curvature form $\Theta\left(\widehat{\nabla}_{E}\right)$ satisfies $\frac{\sqrt{-1}}{2 \pi} \Theta\left(\widehat{\nabla}_{E}\right)=\omega$.

6.10. Proof of vanishing theorems of Kodaira-Akizuki-Nakano, Girbau and Grauert-Riemenschneider. Theorem 1.3 follows from (32) of Lemma 6.6 as in the case of complex manifolds. The proof below is essentially taken from Section 4.9 of Demailly [15].

Proof of Theorem 1.3. We use the notation in Section 6.6. Let $x$ be a point on $M$ and $\phi_{j}: U_{j} \rightarrow B_{j}^{n}$ be a member of the defining 1-cocycle of $\mathcal{F}$ which contains $x$. Let $\omega_{j}$ be a Kähler form on $B_{j}^{n}$ which satisfies $\left.\omega\right|_{U_{j}}=\phi_{j}^{*} \omega_{j}$, where $\omega$ is the transverse Kähler form of $\left(M, \mathcal{F}, J, g_{\nu}\right)$. We can modify $\phi_{j}$ so that $\left(\omega_{j}\right)_{\phi_{j}(x)}=\sum_{i=1}^{n} \sqrt{-1} d z_{i} \wedge$ $d \bar{z}_{i}$ at $\phi_{j}(x)$ by the holomorphic coordinate change of $B_{j}^{n}$. We regard the curvature form $\left(\sqrt{-1} \Theta\left(\nabla_{j}\right)\right)_{\phi_{j}(x)}$ of $E_{j}$ at $\phi_{j}(x)$ as a Hermitian matrix. By diagonalizing this Hermitian matrix $\left(\sqrt{-1} \Theta\left(\nabla_{j}\right)\right)_{\phi_{j}(x)}$ by a unitary matrix, we get a holomorphic 
coordinate $\left(w_{1}, w_{2}, \ldots, w_{n}\right)$ of $B_{j}^{n}$ such that $\omega_{\phi_{j}(x)}=\sum_{i=1}^{n} \sqrt{-1} d w_{i} \wedge d \bar{w}_{i}$ and $\left(\sqrt{-1} \Theta\left(\nabla_{j}\right)\right)_{\phi_{j}(x)}=\sum_{i=1}^{n} \sqrt{-1} \lambda_{i}(x) d w_{i} \wedge d \bar{w}_{i}$ for some real numbers $\lambda_{i}(x)$. The assumption of the positivity of $E$ implies that $\lambda_{i}(x)$ is positive. We reorder $\lambda_{i}(x)$ so that $\lambda_{1}(x) \leq \lambda_{2}(x) \leq \cdots \leq \lambda_{n}(x)$. We consider a vector bundle

$$
E^{p, q}=\bigwedge^{p}\left(T^{*} M / T^{*} \mathcal{F}\right)^{1,0} \otimes \bigwedge^{q}\left(T^{*} M / T^{*} \mathcal{F}\right)^{0,1} \otimes E
$$

over $(M, \mathcal{F})$. Then $E^{p, q}$ is $\mathcal{F}$-fibered, and basic $(p, q)$-forms are regarded as basic sections of $E^{p, q}$. Let

$$
E_{j}^{p, q}=\bigwedge^{p}\left(T^{*} B_{j}^{n}\right)^{1,0} \otimes \bigwedge^{q}\left(T^{*} B_{j}^{n}\right)^{0,1} \otimes E_{j}
$$

be a vector bundle over $B_{j}^{n}$. Then we get $\left.E^{p, q}\right|_{U_{j}}=\phi_{j}^{*} E_{j}^{p, q}$. Let $g^{p, q}$ be the metric on $E^{p, q}$ induced from $g$ and $h_{E}$. Let $g_{j}^{p, q}$ be the metric on $E_{j}^{p, q}$ which satisfies $\phi_{j}^{*} g_{j}^{p, q}=g^{p, q}$. By an argument of linear algebra on $\left(E_{j}^{p, q}\right)_{\phi_{j}(x)}$, we get

$$
g_{j}^{p, q}\left(\left[\sqrt{-1} \Theta\left(\nabla_{j}\right), \Lambda_{j}\right] \alpha_{j}, \alpha_{j}\right) \geq\left(\sum_{i=1}^{\min \{p, q\}} \lambda_{i}(x)-\sum_{i=\max \{p, q\}+1}^{n} \lambda_{i}(x)\right) g_{j}^{p, q}\left(\alpha_{j}, \alpha_{j}\right)
$$

for $\alpha_{j}$ in $\left(E_{j}^{p, q}\right)_{\phi_{j}(x)}$. Then, for a basic $(p, q)$-form $\alpha$ valued in $E$, we get

$$
g_{x}^{p, q}\left(\left[\sqrt{-1} \Theta\left(\nabla_{E}\right), \Lambda\right] \alpha_{x}, \alpha_{x}\right) \geq\left(\sum_{i=1}^{\min \{p, q\}} \lambda_{i}(x)-\sum_{i=\max \{p, q\}+1}^{n} \lambda_{i}(x)\right) g_{x}^{p, q}\left(\alpha_{x}, \alpha_{x}\right)
$$

at each point $x$ on $M$. Note that $\lambda_{j}(x)$ is a leafwise constant continuous function on $M$. Since $\alpha_{x} \widetilde{\wedge} \bar{*}_{b, E} \beta_{x}=g_{x}^{p, q}\left(\alpha_{x}, \beta_{x}\right) \omega^{n}$ at each point $x$ on $M$ for $\alpha$ and $\beta$ in $\Omega_{b}^{p, q}(M / \mathcal{F}, E)$, we get

$$
\left(\left[\sqrt{-1} \Theta\left(\nabla_{E}\right), \Lambda\right] \alpha, \alpha\right) \geq \int_{W}\left(\sum_{i=1}^{q} \lambda_{i}-\sum_{i=p+1}^{n} \lambda_{i}\right) \mathcal{I}\left(\rho^{*}\left(\alpha \widetilde{\wedge}_{\bar{*}_{b, E}} \alpha\right) \wedge \chi\right)
$$

by (35) (see Section 6.4 for the definition of the inner product $(\cdot, \cdot)$ and the right hand side). Since $\left(\Delta_{E}^{\prime \prime} \alpha, \alpha\right)=\left\|\nabla_{E}^{\prime \prime} \alpha\right\|^{2}+\left\|\nabla_{E}^{\prime \prime \star} \alpha\right\|^{2}$ and $\left(\Delta_{E}^{\prime} \alpha, \alpha\right) \geq 0$, the BochnerKodaira-Nakano identity (32) of Lemma 6.6 implies

$$
\left\|\nabla_{E}^{\prime \prime} \alpha\right\|^{2}+\left\|\nabla_{E}^{\prime \prime \star} \alpha\right\|^{2} \geq\left(\left[\sqrt{-1} \Theta\left(\nabla_{E}\right), \Lambda\right] \alpha, \alpha\right) .
$$

By Proposition 6.10, we fix a transverse Kähler metric on $(M, \mathcal{F}, J)$ whose transverse Kähler form is equal to $\sqrt{-1} \Theta\left(\nabla_{E}\right)$. Then $\lambda_{j}(x)=1$ for every $j$ and $x$. We get

$$
\left\|\nabla_{E}^{\prime \prime} \alpha\right\|^{2}+\left\|\nabla_{E}^{\prime \prime} \alpha\right\|^{2} \geq(p+q-n)\|\alpha\|^{2} .
$$

Thus if $\alpha$ is harmonic and $p+q>n$, we get $\alpha=0$.

The analog of Grauert-Riemenschneider's vanishing theorem [25] (Theorem6.11) and Girbau's vanishing theorem 21] (Theorem 6.12) also follow from the BochnerKodaira-Nakano equality as in [14: Let $(M, \mathcal{F})$ be a closed manifold with a homologically orientable transversely Kähler foliation. Let $\left(E, h_{E}\right)$ be a holomorphic $\mathcal{F}$-fibered Hermitian line bundle on $(M, \mathcal{F})$. The rank of $\Theta\left(\nabla_{E}\right)$ at a point $x$ on $M$ is defined by the number of positive eigenvalues as a Hermitian matrix. 
Theorem 6.11. If $\Theta\left(\nabla_{E}\right)$ has rank at least equal to $s$ at some point on $M$, then

$$
H_{b}^{0, q}\left(M / \mathcal{F}, E^{*}\right)=0
$$

for $q<s$.

Theorem 6.12. If $\Theta\left(\nabla_{E}\right)$ has rank at least equal to $s$ at every point on $M$, then

$$
H_{b}^{p, q}\left(M / \mathcal{F}, E^{*}\right)=0
$$

for $p+q<s$.

We omit the proof of Theorems 6.11 and 6.12, which are similar to the argument of Demailly in the proof of [14, Proposition in Section 2].

Remark 6.13. We remark on the other possibility of the inner product on the basic de Rham complex. Álvarez López [3] proved the Hodge decomposition theorem for a basic de Rham complex with respect to the inner product $\langle\langle\cdot, \cdot\rangle\rangle$ defined by the restriction of the usual inner product of the de Rham complex. Note that the formal adjoint of $d$ with respect to $\langle\langle\cdot, \cdot\rangle\rangle$ is not given by the basic Hodge star operator as noted in Remark 6.5. Hence Lemma 6.4 is not true in general for $\langle\langle\cdot, \cdot\rangle\rangle$. To show Theorem 1.3 using $\langle\langle\cdot, \cdot\rangle\rangle$, one can apply Masa's theorem in 34 for the existence of a minimal metric on homologically orientable foliations. Since the mean curvature form is zero for a minimal metric, the adjoint of $d$ with respect to $\langle\langle\cdot, \cdot\rangle\rangle$ is given by its conjugation of $d$ by the basic Hodge operator. Then Lemma 6.4 is true for $\langle\langle\cdot, \cdot\rangle\rangle$. Then the rest of the argument is the same as above.

6.11. Proof of the vanishing theorem for positive Sasakian manifolds. We will prove Theorem 1.2 by Theorem $\left[1.3\right.$, Let $\left(\mathcal{F}, J, g_{\nu}\right)$ be a complex codimension $n$ transversely Kähler foliation on a closed manifold $M$. We recall

Definition 6.14. A holomorphic $\mathcal{F}$-fibered Hermitian line bundle

$$
K_{\mathcal{F}}=\bigwedge^{n}\left(T^{*} M / T^{*} \mathcal{F}\right)^{1,0}
$$

on $M$ is called the canonical line bundle of $(M, \mathcal{F}, J)$. The dual of the canonical line bundle of $(M, \mathcal{F}, J)$ is called the anticanonical line bundle of $(M, \mathcal{F}, J)$. A Sasakian manifold is called positive if the anticanonical line bundle of the underlying transversely Kähler flow is positive.

We refer to Boyer, Galicki and Nakamaye [10] for more detailed information on positive Sasakian manifolds.

We assume the homological orientability of $\mathcal{F}$ to apply the results in this section. Let $K_{\mathcal{F}}$ be the canonical line bundle of $(M, \mathcal{F})$. We fix a transverse Hermitian metric on $(T M / T \mathcal{F}) \otimes \mathbb{C}$. Let $h_{K_{\mathcal{F}}}$ be the Hermitian metric on $K_{\mathcal{F}}$ induced from the metric on $(T M / T \mathcal{F}) \otimes \mathbb{C}$. We recall a $\mathbb{C}$-antilinear isomorphism

$$
h: K_{\mathcal{F}} \longrightarrow K_{\mathcal{F}}^{*}
$$

defined by $h(s)=h_{K_{\mathcal{F}}}(s, \cdot)$. Note that the canonical line bundle $\left(K_{\mathcal{F}}, h_{K_{\mathcal{F}}}\right)$ of $\mathcal{F}$ is a holomorphic $\mathcal{F}$-fibered Hermitian line bundle.

Let $\mathbf{1}_{K_{\mathcal{F}}}$ be the identity in $\operatorname{End}\left(K_{\mathcal{F}}\right)=C^{\infty}\left(K_{\mathcal{F}} \otimes K_{\mathcal{F}}^{*}\right)$. We have natural maps

$$
\begin{aligned}
& \Xi_{1}: \Omega_{b}^{0, q}(M / \mathcal{F}) \longrightarrow \Omega_{b}^{n, q}\left(M / \mathcal{F}, K_{\mathcal{F}}^{*}\right), \\
& \Xi_{2}: \Omega_{b}^{n, q}(M / \mathcal{F}) \longrightarrow \Omega_{b}^{0, q}\left(M / \mathcal{F}, K_{\mathcal{F}}\right)
\end{aligned}
$$


defined by $\Xi_{1}(\alpha)=\alpha \wedge \mathbf{1}_{K_{\mathcal{F}}}$ and $\Xi_{2}(\beta \wedge \alpha)=\alpha \otimes \beta$ for $\beta$ in $\Omega_{b}^{0, q}(M / \mathcal{F})$ and $\beta$ in $\Omega_{b}^{n, 0}(M / \mathcal{F})=C^{\infty}\left(K_{\mathcal{F}}\right)$. Clearly $\Xi_{1}$ and $\Xi_{2}$ are bijective.

Let $\mathbf{H}_{b}^{p, q}$ be the space of basic harmonic $(p, q)$-forms. Let $\mathbf{H}_{b}^{p, q}\left(K_{\mathcal{F}}^{*}\right)$ be the space of basic harmonic $(p, q)$-forms with values in $K_{\mathcal{F}}^{*}$.

Lemma 6.15.

$$
\Xi_{1}\left(\mathbf{H}_{b}^{0, q}\right)=\mathbf{H}_{b}^{n, q}\left(K_{\mathcal{F}}^{*}\right)
$$

Proof. It is easy to see

$$
\begin{aligned}
& \Xi_{1} \bar{\partial}=\nabla_{K_{\mathcal{F}}^{*}}^{\prime \prime} \Xi_{1}, \\
& \Xi_{2} \bar{\partial}=\nabla_{K_{\mathcal{F}}}^{\prime \prime} \Xi_{2},
\end{aligned}
$$

where (36) follows from the fact that $\mathbf{1}_{K_{\mathcal{F}}}$ is a holomorphic section of $K_{\mathcal{F}} \otimes K_{\mathcal{F}}^{*}$. By (36) and the bijectivity of $\Xi_{1}$, we get

$$
\Xi_{1}(\operatorname{ker} \bar{\partial})=\operatorname{ker} \nabla_{K_{\mathcal{F}}^{*}}^{\prime \prime}
$$

Let $s$ be a local basic section of $K_{\mathcal{F}}$ such that $h(s, s)=1$. It is easy to see

$$
s \otimes h(s)=\mathbf{1}_{K_{\mathcal{F}}} .
$$

By the definition of $\bar{*}_{b}$ and $h(s, s)=1$, we have

$$
\bar{*}_{b} \alpha=(-1)^{n q} s \wedge \bar{*}_{b}(s \wedge \alpha) .
$$

Thus we get

$$
\begin{aligned}
\bar{*}_{b, K_{\mathcal{F}}^{*}} \Xi_{1}(\alpha) & =\bar{*}_{b, K_{\mathcal{F}}^{*}}\left(\alpha \wedge \mathbf{1}_{K_{\mathcal{F}}}\right) \\
& =\bar{\star}_{b, K_{\mathcal{F}}^{*}}(\alpha \wedge s \otimes h(s)) \\
& =\bar{*}_{b}(\alpha \wedge s) \otimes s \\
& =\Xi_{2}\left(s \wedge \bar{*}_{b}(\alpha \wedge s)\right) \\
& =\Xi_{2} \bar{*}_{b} \alpha .
\end{aligned}
$$

By substituting $\bar{*}_{b} \beta$ to $\alpha$ in (39) and composing $\bar{*}_{b, K_{\mathcal{F}}}$ to both sides of the equation, we get

$$
\Xi_{1} \bar{*}_{b}=\bar{*}_{b, K_{\mathcal{F}}} \Xi_{2} .
$$

By (29), we get $\bar{\partial}^{\star}=-\bar{*}_{b} \bar{\partial}_{b}$. Hence, by equations (37), (39) and (40), we get

$$
\Xi_{1} \bar{\partial}^{\star}=-\Xi_{1} \bar{*}_{b} \bar{\partial} \bar{*}_{b}=-\bar{*}_{b, K_{\mathcal{F}}} \nabla_{K_{\mathcal{F}}}^{\prime \prime} \bar{*}_{b, K_{\mathcal{F}}^{*}} \Xi_{1}=\nabla_{K_{\mathcal{F}}^{*}}^{\prime \prime \star} \Xi_{1} .
$$

By (41) and the bijectivity of $\Xi_{1}$, we get

$$
\Xi_{1}\left(\operatorname{ker} \bar{\partial}^{\star}\right)=\operatorname{ker} \nabla_{K_{\mathcal{F}}^{*}}^{\prime \prime}
$$

Since $\mathbf{H}_{b}^{0, q}=\operatorname{ker} \bar{\partial} \cap \operatorname{ker} \bar{\partial}^{\star}$ and $\mathbf{H}_{b}^{n, q}\left(K_{\mathcal{F}}^{*}\right)=\operatorname{ker} \nabla_{K_{\mathcal{F}}^{*}}^{\prime \prime} \cap \operatorname{ker} \nabla_{K_{\mathcal{F}}^{*}}^{\prime \prime \star}$ by the Hodge decomposition theorem of El Kacimi Alaoui (see Theorem 6.7), the proof is done by (38) and (42).

Remark 6.16. We can prove $\Xi_{2}\left(\mathbf{H}_{b}^{n, q}\right)=\mathbf{H}_{b}^{0, q}\left(K_{\mathcal{F}}\right)$ similarly.

Lemma 6.15 allows us to prove Theorem 1.2 by Theorem 1.3 without using the sheaf cohomology of basic holomorphic forms. 
Proof of Theorem 1.2. It is known that the Reeb flow of a Sasakian manifold is homologically orientable as we saw in Example 6.2. Let $\mathbf{H}_{b}^{p, q}$ be the space of $\Delta_{\bar{\partial}^{-}}$ harmonic basic $(p, q)$-forms on $(M, \mathcal{F})$. By the Kähler identity for homologically orientable transversely Kähler foliations by El Kacimi Alaoui (Proposition 3.4.5 of [19] $), \mathbf{H}_{b}^{p, q}$ is equal to the space of $\Delta_{d}$-harmonic $(p, q)$-forms, where $\Delta_{d}=\left[d, d^{\star}\right]$. Since $\Delta_{d}$ commutes with the complex conjugation, we get

$$
\mathbf{H}_{b}^{p, 0} \cong \mathbf{H}_{b}^{0, p}
$$

by the complex conjugation. By Lemma 6.15, we get

$$
\mathbf{H}_{b}^{0, p} \cong \mathbf{H}_{b}^{n, p}\left(K_{\mathcal{F}}^{*}\right),
$$

where $\mathbf{H}_{b}^{n, p}\left(K_{\mathcal{F}}^{*}\right)$ is the space of $\Delta_{K_{\mathcal{F}}^{*}}^{\prime \prime}$-harmonic basic $(n, p)$-forms on $(M, \mathcal{F})$ valued in $K_{\mathcal{F}}^{*}$. Since $K_{\mathcal{F}}^{*}$ is positive, Theorem 1.3 implies that $\mathbf{H}_{b}^{n, p}\left(K_{\mathcal{F}}^{*}\right)$ vanishes for $p>0$.

\section{Moduli SPACE of SASAKian MEtrics \\ WITH A FIXED UNDERLYING TRANSVERSE KÄHLER FLOW}

We will prove Theorem 1.6

Let $M$ be a closed manifold with a Sasakian metric with contact form $\eta$. Let $\mathfrak{k}_{0}$ be the underlying transversely Kähler flow. Recall that Diff ${ }_{0}\left(\mathfrak{k}_{0}\right)$ is the identity component of the subgroup of $\operatorname{Diff}(M)$ consisting of diffeomorphisms which preserve $\mathfrak{k}_{0}$, and $\operatorname{Ham}\left(\mathfrak{k}_{0}\right)$ is defined in (1). Obviously we have an exact sequence

$$
0 \longrightarrow \operatorname{Ham}\left(\mathfrak{k}_{0}\right) \longrightarrow \operatorname{Diff}_{0}\left(\mathfrak{k}_{0}\right) \stackrel{\Pi}{\longrightarrow} H^{1}(M ; \mathbb{R}),
$$

where $\Pi$ is defined by $\Pi(f)=\left[\eta-f^{*} \eta\right]$ for $f$ in $\operatorname{Diff}_{0}\left(\mathfrak{k}_{0}\right)$. Note that $\eta-f^{*} \eta$ is closed, because $f$ preserves the transverse Kähler form $d \eta$.

Recall that we denote the subgroup of $\operatorname{Diff}(M)$ consisting of diffeomorphisms which map each leaf of $\mathcal{F}$ to itself by $\operatorname{Diff}(M, \mathcal{F})$. The identity component of $\operatorname{Diff}(M, \mathcal{F})$ with respect to the Fréchet topology is denoted by $\operatorname{Diff}_{0}(M, \mathcal{F})$. Note that $\operatorname{Diff}_{0}(M, \mathcal{F})$ is a subgroup of $\operatorname{Ham}\left(\mathfrak{k}_{0}\right)$.

Lemma 7.1. Let $\xi$ be a nowhere vanishing vector field on a closed manifold $M$ which is tangent to a flow $\mathcal{F}$. Let $\eta$ be a 1-form on $M$ which satisfies $\mathcal{L}_{\xi} \eta=0$ and $\eta(\xi)=1$. Let $h$ be a leafwise constant smooth function on $(M, \mathcal{F})$. Then there exists $f$ in $\operatorname{Diff}_{0}(M, \mathcal{F})$ such that

$$
f^{*} \eta=\eta+d h .
$$

Proof. Let $\left\{\varphi_{s}\right\}_{s \in \mathbb{R}}$ be the flow generated by $\xi$. We consider maps

$$
\begin{aligned}
\Gamma: \quad M \times \mathbb{R} & \longrightarrow M \times \mathbb{R}, \\
(x, s) & \longmapsto(x, h(x) s)
\end{aligned}
$$

and

Let

$$
\begin{aligned}
\Psi: \quad M \times \mathbb{R} & \longrightarrow M \times \mathbb{R}, \\
(x, s) & \longmapsto\left(\varphi_{s}(x), s\right) .
\end{aligned}
$$

$$
f=\left.\operatorname{pr}_{1} \circ \Psi \circ \Gamma\right|_{M \times\{1\}},
$$

where $\operatorname{pr}_{1}: M \times \mathbb{R} \longrightarrow M$ is the first projection. The differential map $T f$ of $f$ at $(x, s)$ is

$$
T f=T\left(\left.\operatorname{pr}_{1} \circ \Psi \circ \Gamma\right|_{M \times\{1\}}\right)=T \varphi_{1}+s(d h \otimes \xi) .
$$


Then $T \varphi_{1}+s d h \otimes \xi$ is nondegenerate, because $h$ is constant on each leaf of $\mathcal{F}$. So $f$ is an open immersion. Since an open immersion from a closed manifold to a closed manifold of degree 1 is a diffeomorphism, $f$ is a diffeomorphism of $M$. By (44), we have

$$
f^{*} \eta(Y)=\eta\left(\left(T \varphi_{1}+d h \otimes \xi\right)(Y)\right)=\varphi_{1}^{*} \eta(Y)+d h(Y)=\eta(Y)+d h(Y)
$$

for a vector field $Y$ on $M$. Thus $f$ satisfies equation (43).

Recall that a Sasakian metric on $M$ is determined by a contact form $\eta$ whose Reeb flow has a transversely Kähler structure such that $d \eta$ is the transverse Kähler form (Proposition 2.16). Let $(M, \eta, g)$ be a closed Sasakian manifold. We denote the Reeb flow of $\eta$ by $\mathcal{F}$. We denote the underlying transversely Kähler foliation by $\mathfrak{k}_{0}$. For a basic closed 1 -form $\beta$ on $(M, \mathcal{F})$, we can construct a Sasakian metric $\sigma_{\beta}$ determined by a contact form $\eta+\beta$ and the same transversely Kähler structure of $\mathcal{F}$ as $(\eta, g)$. We consider the set of Sasakian metrics

$$
\mathcal{S}_{1}=\left\{\sigma_{\beta} \mid \beta \in \mathbf{H}_{b}^{1}\right\},
$$

where $\mathbf{H}_{b}^{1}$ is the space of basic harmonic 1-form on $(M, \mathcal{F})$.

Proposition 7.2. Every orbit of the action of $\operatorname{Ham}\left(\mathfrak{k}_{0}\right)$ on $\mathcal{S}$ intersects $\mathcal{S}_{1}$.

Proof. Take a Sasakian metric $\left(\eta_{1}, g_{1}\right)$ whose underlying transversely Kähler flow is isomorphic to $\mathfrak{k}_{0}$. The isomorphism of transversely Kähler flows means $d \eta=d \eta_{1}$ and the transverse metrics on $\mathcal{F}$ induced by $g$ and $g_{1}$ are equal. By Lemma 3.17 there exist a real number $r$ and a diffeomorphism $f_{1}$ of $M$ in $\operatorname{Diff}_{0}(M, \mathcal{F})$ such that

$$
\left.\eta\right|_{T \mathcal{F}}=r f_{1}^{*}\left(\left.\eta_{1}\right|_{T \mathcal{F}}\right) .
$$

Let $\eta_{2}=f_{1}^{*} \eta_{1}$. Since $\left.\left(\eta-r \eta_{2}\right)\right|_{T \mathcal{F}}=0$ and $d\left(\eta-r \eta_{2}\right)$ is basic, $\eta-r \eta_{2}$ is basic. Hence we have

$$
[d \eta]=r\left[d \eta_{2}\right]=r\left[d \eta_{1}\right]
$$

in $H_{b}^{2}(M / \mathcal{F})$. Note that $\operatorname{Diff}_{0}(M, \mathcal{F})$ trivially acts on the basic forms by the definition. Since we have $\left[d \eta_{1}\right]=[d \eta]$ by the assumption, we have $r=1$. Thus $\eta-\eta_{2}$ is a closed basic 1-form. By the basic Hodge decomposition for Riemannian foliations (see El Kacimi Alaoui and Hector [20] or Álvarez López [3]), there exists a basic harmonic 1-form $\beta$ and a smooth basic function $h_{2}$ such that

$$
\eta-\eta_{2}=\beta+d h_{2} .
$$

Let $\xi$ be the common Reeb vector field of $\eta$ and $\eta_{2}$. By Lemma 7.1 we have a diffeomorphism $f$ of $M$ in $\operatorname{Diff}_{0}(M, \mathcal{F})$ such that

$$
f_{2}^{*} \eta_{2}=\eta_{2}+d h_{2}
$$

Hence we have

$$
f_{2}^{*} \eta_{2}=\eta-\beta
$$

Thus $f_{2}^{*} \eta_{2}$ belongs to $\mathcal{S}_{1}$. Since $f_{2}^{*} \eta_{2}$ is on the same orbit as the action of $\operatorname{Diff} 0(M, \mathcal{F})$ as $\eta_{1}$, it follows that $\mathcal{S}_{1}$ intersects with every orbit on the action of $\operatorname{Diff}_{0}(M, \mathcal{F})$.

Proposition 7.3. Every orbit on the action of $\operatorname{Ham}\left(\mathfrak{k}_{0}\right)$ on $\mathcal{S}$ intersects $\mathcal{S}_{1}$ in at most one point. 
Proof. Assume that we have

$$
\eta-\beta_{1}=f^{*}\left(\eta-\beta_{2}\right)
$$

for $\beta_{1}, \beta_{2}$ in $\mathbf{H}_{b}^{1}$ and $f$ in $\operatorname{Ham}\left(\mathfrak{k}_{0}\right)$. We will show $\beta_{1}=\beta_{2}$.

Here $f^{*} \beta_{2}$ is a basic harmonic 1 -form cohomologous to $\beta_{2}$, because $f$ is isotopic to the identity and preserves the transverse metric of $\mathcal{F}$. Since each basic cohomology class is represented by a unique harmonic form by the Hodge decomposition theorem for basic cohomology (see El Kacimi Alaoui and Hector [20] or Álvarez López [3]), we have

$$
f^{*} \beta_{2}=\beta_{2}
$$

We take an isotopy $\left\{\varphi_{s}\right\}_{s \in[0,1]}$ such that $\varphi_{s}$ belongs to $\operatorname{Ham}_{0}\left(\mathfrak{k}_{0}\right)$ for every $s$ in $[0,1]$, and $\varphi_{0}=\mathrm{id}$ and $\varphi_{1}=f$. Let $X_{s}=\left.\frac{d}{d t}\right|_{u=s} \varphi_{u}$. Since $\eta-\varphi_{s}^{*} \eta$ is exact, $\mathcal{L}_{X_{s}} \eta=\iota_{X_{s}} d \eta+d\left(\eta\left(X_{s}\right)\right)$ is exact. Thus we have a smooth function $h_{s}$ on $M$ such that

$$
\iota_{X_{s}} d \eta=d h_{s}
$$

for each $s$ in $[0,1]$. Since $\mathcal{L}_{h \xi} \eta=d h$ for any smooth function $h$ on $M$, (47) implies

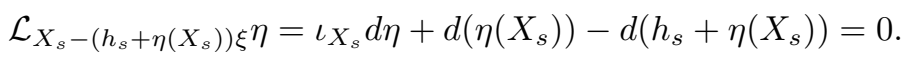

Let $\left\{\varphi_{s}^{\prime}\right\}_{s \in[0,1]}$ be the isotopy generated by vector fields $\left\{X_{s}+\left(h_{s}-\eta\left(X_{s}\right)\right) \xi\right\}_{s \in[0,1]}$. We have

$$
\left(\varphi_{1}^{\prime}\right)^{*} \eta=\eta
$$

by (48). Letting $f_{3}=\left(\varphi_{1}^{\prime}\right)^{-1} \circ f$, we have

$$
f^{*} \eta=f_{3}^{*}\left(\varphi_{1}^{\prime}\right)^{*} \eta=f_{3}^{*} \eta
$$

by (49). The difference of the two vector fields which generate $\left\{\varphi_{s}\right\}_{s \in[0,1]}$ and $\left\{\varphi_{s}^{\prime}\right\}_{s \in[0,1]}$ is $\left(h_{s}+\eta\left(X_{s}\right)\right) \xi$, which is tangent to $\mathcal{F}$. Thus $\varphi_{s}$ and $\varphi_{s}^{\prime}$ induce the same map on the leaf space of $(M, \mathcal{F})$. Thus $\left\{\left(\varphi_{s}^{\prime}\right)^{-1} \circ \varphi_{s}\right\}_{s \in[0,1]}$ gives an isotopy such that $\left(\varphi_{0}^{\prime}\right)^{-1} \circ \varphi_{0}=\mathrm{id},\left(\varphi_{1}^{\prime}\right)^{-1} \circ \varphi_{1}=f_{3}$ and $\left(\varphi_{s}^{\prime}\right)^{-1} \circ \varphi_{s}$ belongs to $\operatorname{Diff}_{0}(M, \mathcal{F})$. Let $\psi_{s}=\left(\varphi_{s}^{\prime}\right)^{-1} \circ \varphi_{s}$. Let $Y_{s}=\left.\frac{d}{d u}\right|_{u=s} \psi_{u}$. Since $\psi_{s}$ belongs to $\operatorname{Diff}_{0}(M, \mathcal{F})$, this $Y_{s}$ is tangent to $\mathcal{F}$ for each $s$. Hence we have

$$
\iota_{Y_{s}} d \eta=0 .
$$

By (51), we have

$$
\begin{aligned}
f_{3}^{*} \eta-\eta=\left.\int_{0}^{1} \frac{d}{d u}\right|_{u=s}\left(\psi_{u}^{*} \eta\right) d s= & \int_{0}^{1} \psi_{s}^{*}\left(\mathcal{L}_{Y_{s}} \eta\right) d s \\
& =\int_{0}^{1} \psi_{s}^{*} d\left(\eta\left(Y_{s}\right)\right) d s=d\left(\int_{0}^{1} \psi_{s}^{*}\left(\eta\left(Y_{s}\right)\right) d s\right) .
\end{aligned}
$$

Hence, letting $h_{3}=\int_{0}^{1} \psi_{u}^{*}\left(\eta\left(Y_{s}\right)\right) d u$, we have

$$
f_{3}^{*} \eta-\eta=d h_{3}
$$

By (45), (46), (50) and (52), we have

$$
\beta_{2}-\beta_{1}=d h_{3}
$$

Since the right hand side is a harmonic form, we have $\beta_{1}=\beta_{2}$.

Theorem 1.6 follows from Propositions 7.2 and 7.3 
Remark 7.4. We remark that we obtain the following result by the proof of Proposition 7.2

Proposition 7.5. Let $\eta_{1}$ and $\eta_{2}$ be the underlying contact forms on a closed manifold $M$ of two $K$-contact structures. If $H^{1}(M ; \mathbb{R})=0$ and the Reeb flows of $\eta_{1}$ and $\eta_{2}$ are equal, then there exists a real number $r$ and a diffeomorphism $f: M \rightarrow M$ such that $r f^{*} \eta_{2}=\eta_{1}$.

\section{EXAMPLES}

8.1. Standard Sasakian metrics on spheres. We recall the standard Sasakian metric on $S^{2 n-1}$. Consider a function $r$ on $\mathbb{R}^{2 n}$ defined by

$$
r\left(x_{1}, y_{1}, x_{2}, y_{2}, \ldots, x_{n}, y_{n}\right)=\sqrt{x_{1}^{2}+y_{1}^{2}+x_{2}^{2}+y_{2}^{2}+\cdots+x_{n}^{2}+y_{n}^{2}},
$$

where $\left(x_{1}, y_{1}, x_{2}, y_{2}, \ldots, x_{n}, y_{n}\right)$ is the standard coordinate on $\mathbb{R}^{2 n}$. Let $S^{2 n-1}=$ $r^{-1}(1)$ be the unit sphere of $\mathbb{R}^{2 n}$. We consider the standard metric $g_{\text {std }}$ and the standard contact form of $\eta_{\text {std }}$ on $\mathbb{R}^{2 n}-\{0\}$ defined by

$$
g_{\mathrm{std}}=\sum_{i=1}^{n}\left(d x_{i} \otimes d x_{i}+d y_{i} \otimes d y_{i}\right), \quad \eta_{\mathrm{std}}=\frac{1}{2 r\left(x_{1}, \ldots, y_{n}\right)^{2}} \sum_{i=1}^{n}\left(x_{i} d y_{i}-y_{i} d x_{i}\right) .
$$

Then $\left(S^{2 n-1},\left.g_{\mathrm{std}}\right|_{S^{2 n-1}},\left.\eta_{\mathrm{std}}\right|_{S^{2 n-1}}\right)$ is a Sasakian manifold by definition, because the Kähler manifold $\left(\mathbb{R}^{2 n}-\{0\}, g_{\text {std }}, d \eta_{\text {std }}\right)$ is isomorphic to $\left(S^{2 n-1} \times \mathbb{R}_{>0}, d r^{2}+\right.$ $\left.\left.r^{2} g_{\text {std }}\right|_{S^{2 n-1}}, d\left(\left.r^{2} \eta_{\text {std }}\right|_{S^{2 n-1}}\right)\right)$.

The flow generated by the Reeb vector field of $\left.\eta_{\mathrm{std}}\right|_{S^{2 n-1}}$ is given by the principal $S^{1}$-action whose orbits are tangent to the fiber of Hopf fibration. The base space of the Hopf fibration is $\mathbb{C} P^{n-1}$.

To describe the deformation of transversely holomorphic flows, we use the following

Proposition 8.1 (Girbau, Haefliger and Sundararaman [22, Proposition 6.1]). The Kuranishi space of the deformation of the transversely holomorphic flow defined by fibers of a circle bundle over a complex manifold $X$ is identified with an open neighborhood of 0 in $H^{0}\left(X, T^{1,0} X\right)$, the space of holomorphic vector fields on $X$, if $X$ satisfies both of the following:

$$
H^{1,0}(X)=0, H^{1}\left(X, T^{1,0} X\right)=0 .
$$

Here $\mathbb{C} P^{n-1}$ satisfies $H^{1,0}\left(\mathbb{C} P^{n-1}\right)=0$ and $H^{1}\left(\mathbb{C} P^{n-1}, T^{1,0} \mathbb{C} P^{n-1}\right)=0$. Hence Proposition 8.1 implies that the Kuranishi space as a transversely holomorphic flow is identified with an open neighborhood of 0 in $H^{0}\left(\mathbb{C} P^{n-1}, T^{1,0} \mathbb{C} P^{n-1}\right)$, which is of complex dimension $n^{2}-1$. Among them, infinitesimal deformation of transversely holomorphic Riemannian flows form a union of real vector subspaces of real dimension $n-1$.

Since $H^{0,2}\left(\mathbb{C} P^{n-1}\right)=0$, Corollary 1.4 implies that the Sasakian metric $\left(g_{\text {std }}, \eta_{\text {std }}\right)$ is stable, that is, a family of compatible Sasakian metric exists for any small deformation of the Reeb flow as transversely holomorphic Riemannian flows. Since $S^{2 n-1}$ is simply connected, Corollary 1.7 implies that the space of isomorphism classes of Sasakian metrics are identified with the isomorphism classes of the underlying transversely Kähler flows. 
We can apply Proposition 8.1 and Corollaries 1.4 and 1.7 to a Sasakian metric on the circle bundles associated to positive line bundles over Fano manifolds $X$ which satisfies $H^{1}\left(X, T^{1,0} X\right)=0$ in a similar way.

Remark 8.2. We note that deformation of the Hopf fibration as transversely holomorphic foliations is studied by Duchamp and Kalka [17] and Haefliger [27. Other results related to Proposition 8.1 is obtained by Boyer and Galicki in [8, Section 8.2.2].

8.2. Circle bundles over complex tori. We present an example of deformations of the Reeb flow of a Sasakian manifold which does not admit compatible Sasakian metrics.

Let $X$ be a projective complex torus with a positive holomorphic line bundle $E$. We fix a Hermitian metric on $E$ so that its curvature form is positive. Let $M$ be the unit circle bundle of $E$. Then $M$ has a Sasakian metric whose underlying transversely Kähler flow is defined by the fibers of the $S^{1}$-bundle by the BoothbyWang construction due to Hatakeyama [28, Corollary of Theorem 4]).

It is well known that there exists a smooth family of complex tori $\left\{X^{t}\right\}_{t \in]-1,1[}$ and a dense subset $K$ in $]-1,1\left[\right.$ such that $X^{0}=X$ and $X^{t}$ is not projective for every $t$ in $K$. It is easy to see that every complex torus is Kähler. So $X^{t}$ is Kähler.

We denote the total space of the family of complex tori by $\mathcal{T}$. We fix a trivialization $\theta: \mathcal{T} \cong X \times]-1,1[$ as a smooth fiber bundle over ] $-1,1[$. We pull back the complex Hermitian line bundle $E$ on $X$ to $\mathcal{T}$ by $\operatorname{pr}_{1} \circ \theta$, where $\left.\operatorname{pr}_{1}: X \times\right]-1,1[\rightarrow X$ is the first projection. We define $M^{t}$ as the unit circle bundle associated to the complex line bundle $\left.\left(\theta^{*} \operatorname{pr}_{1}^{*} E\right)\right|_{X^{t}} \rightarrow X^{t}$. Let $\mathcal{F}^{t}$ be a flow on $M^{t}$ defined by the fibers of the circle bundle $M^{t} \rightarrow X^{t}$. Since the leaf space $X^{t}$ is Kähler, $\mathcal{F}^{t}$ is a transversely Kähler flow for $t$ sufficiently close to 0 .

There exists a compatible Sasakian metric on $M^{0}$ by definition. But, for $t$ in $K$, $M^{t}$ does not have any compatible Sasakian metric. Indeed, if $M^{t}$ has a compatible Sasakian metric, then $X^{t}$ must be projective by Theorem 4 of Hatakeyama [28]. This is a contradiction.

In this example, the basic Euler class of $\mathcal{F}^{t}$ is the Euler class of circle bundles $M^{t} \rightarrow X^{t}$ and can be considered as an element of $H^{2}\left(X^{t} ; \mathbb{Z}\right)$. Clearly this class is of a topological nature and independent of $t$. On the other hand, the Hodge decomposition $H^{2}\left(X^{t} ; \mathbb{C}\right) \cong H^{2,0}\left(X^{t}\right) \oplus H^{1,1}\left(X^{t}\right) \oplus H^{0,2}\left(X^{t}\right)$ changes when $t$ varies.

\section{ACKNOWLEDGEMENT}

The author is grateful to Jesús Antonio Álvarez López for pointing out a mistake in the previous version of this manuscript. It was in the discussion with him during the author's stay at the University of Santiago de Compostela in the spring of 2009. The author would also like to express his deep gratitude to Jesús Antonio Álvarez López for his invitation, great hospitality and valuable discussions. The author would like to thank the referee for a comment on the simplification of the proof of Lemma 6.15.

\section{REFERENCES}

[1] Y. Akizuki and S. Nakano, Note on Kodaira-Spencer's proof of Lefschetz theorems, Proc. Japan Acad. 30 (1954), 266-272. MR0066694(16,619a) 
[2] J. A. Alvarez López, On Riemannian foliations with minimal leaves, Ann. Inst. Fourier (Grenoble) 40 (1990), no. 1, 163-176 (English, with French summary). MR.1056780 (92a:53038)

[3] J. A. Alvarez López, The basic component of the mean curvature of Riemannian foliations, Ann. Global Anal. Geom. 10 (1992), no. 2, 179-194, DOI 10.1007/BF00130919. MR.1175918 (93h:53027)

[4] W. L. Baily, On the imbedding of $V$-manifolds in projective space, Amer. J. Math. 79 (1957), 403-430. MR0100104(20 \#6538)

[5] F. A. Belgun, On the metric structure of non-Kähler complex surfaces, Math. Ann. 317 (2000), no. 1, 1-40, DOI 10.1007/s002080050357. MR1760667 (2002c:32027)

[6] D. E. Blair, Riemannian geometry of contact and symplectic manifolds, Progress in Mathematics, vol. 203, Birkhäuser Boston Inc., Boston, MA, 2002. MR1874240 (2002m:53120)

[7] C. P. Boyer and K. Galicki, Sasakian geometry, hypersurface singularities, and Einstein metrics, Rend. Circ. Mat. Palermo (2) Suppl. 75 (2005), 57-87. MR2152356 (2006i:53065)

[8] C. P. Boyer and K. Galicki, Sasakian geometry, Oxford Mathematical Monographs, Oxford University Press, Oxford, 2008. MR2382957 (2009c:53058)

[9] C. P. Boyer, K. Galicki, and M. Nakamaye, On the geometry of Sasakian-Einstein 5-manifolds, Math. Ann. 325 (2003), no. 3, 485-524, DOI 10.1007/s00208-002-0388-3. MR.1968604 (2004b:53061)

[10] C. P. Boyer, K. Galicki, and M. Nakamaye, On positive Sasakian geometry, Geom. Dedicata 101 (2003), 93-102, DOI 10.1023/A:1026363529906. MR2017897(2005a:53072)

[11] A. Candel and L. Conlon, Foliations. I, Graduate Studies in Mathematics, vol. 23, American Mathematical Society, Providence, RI, 2000. MR.1732868 (2002f:57058)

[12] Y. Carrière, Flots riemanniens, Astérisque 116 (1984), 31-52 (French). Transversal structure of foliations (Toulouse, 1982). MR755161 (86m:58125a)

[13] C. van Coevering, Stability of Sasaki-extremal metrics under complex deformations, to appear in Internat. Math. Res. Notices, DOI 10.1093/imrn/rns210

[14] J.-P. Demailly, Transcendental proof of a generalized Kawamata-Viehweg vanishing theorem, (Cetraro, 1989), Sem. Conf., vol. 8, EditEl, Rende, 1991, pp. 81-94. MR1222208(94j:32025)

[15] J.-P. Demailly, Multiplier ideal sheaves and analytic methods in algebraic geometry, Geometry (Trieste, 2000), ICTP Lect. Notes, vol. 6, Abdus Salam Int. Cent. Theoret. Phys., Trieste, 2001, pp. 1-148. MR1919457 (2003f:32020)

[16] J.-P. Demailly, Complex analytic and differential geometry, Preprint (2009) available at http://www-fourier.ujf-grenoble.fr/ demailly/manuscripts/agbook.pdf

[17] T. Duchamp and M. Kalka, Holomorphic foliations and deformations of the Hopf foliation, Pacific J. Math. 112 (1984), no. 1, 69-81. MR739141 (85m:57019)

[18] A. El Kacimi-Alaoui, Sur la cohomologie feuilletée, Compositio Math. 49 (1983), no. 2, 195215 (French). MR704391 (85a:57016)

[19] A. El Kacimi-Alaoui, Opérateurs transversalement elliptiques sur un feuilletage riemannien et applications, Compositio Math. 73 (1990), no. 1, 57-106 (French, with English summary). MR.1042454 (91f:58089)

[20] A. El Kacimi-Alaoui and G. Hector, Décomposition de Hodge basique pour un feuilletage riemannien, Ann. Inst. Fourier (Grenoble) 36 (1986), no. 3, 207-227 (French, with English summary). MR865667(87m:57029)

[21] J. Girbau, Sur le théorème de Le Potier d'annulation de la cohomologie, C. R. Acad. Sci. Paris Sér. A-B 283 (1976), no. 6, Aiv, A355-A358. MR0430333 (55 \#3338)

[22] J. Girbau, A. Haefliger, and D. Sundararaman, On deformations of transversely holomorphic foliations, J. Reine Angew. Math. 345 (1983), 122-147, DOI 10.1515/crll.1983.345.122. $\operatorname{MR} 717890(84 \mathrm{j}: 32026)$

[23] X. Gómez-Mont, Transversal holomorphic structures, J. Differential Geom. 15 (1980), no. 2 , 161-185 (1981). MR614365 (82j:53065)

[24] R. Goto, Calabi-Yau structures and Einstein-Sasakian structures on crepant resolutions of isolated singularities, J. Math. Soc. Japan 64, no. 3 (2012), 1005-1052.

[25] H. Grauert and O. Riemenschneider, Kählersche Mannigfaltigkeiten mit hyper-q-konvexem Rand, Problems in analysis (Lectures Sympos. in honor of Salomon Bochner, Princeton Univ., Princeton, N.J., 1969), Princeton Univ. Press, Princeton, N.J., 1970, pp. 61-79 (German). MR0355107(50 \#7584) 
[26] A. Haefliger, Some remarks on foliations with minimal leaves, J. Differential Geom. 15 (1980), no. 2, 269-284 (1981). MR614370 (82j:57027)

[27] A. Haefliger, Deformations of transversely holomorphic flows on spheres and deformations of Hopf manifolds, Compositio Math. 55 (1985), no. 2, 241-251. MR795716 (87a:57032)

[28] Y. Hatakeyama, Some notes on differentiable manifolds with almost contact structures, Tohoku Math. J. (2) 15 (1963), 176-181. MR0150718 (27 \#705)

[29] G. Hector, E. Macías, and M. Saralegi, Lemme de Moser feuilleté et classification des variétés de Poisson régulières, Publ. Mat. 33 (1989), no. 3, 423-430, DOI 10.5565/PUBLMAT_33389_04 (French, with English summary). MR_1038481 (91a:58145)

[30] F. W. Kamber and P. Tondeur, Duality for Riemannian foliations, Singularities, Part 1 (Arcata, Calif., 1981), Proc. Sympos. Pure Math., vol. 40, Amer. Math. Soc., Providence, RI, 1983, pp. 609-618. MR 713097 (85e:57030)

[31] S. Kobayashi and K. Nomizu, Foundations of differential geometry. Vol. I, Wiley Classics Library, John Wiley \& Sons Inc., New York, 1996. Reprint of the 1963 original; A WileyInterscience Publication. MR1393940 (97c:53001a)

[32] K. Kodaira and D. C. Spencer, On deformations of complex analytic structures. I, II, Ann. of Math. (2) 67 (1958), 328-466. MR0112154 (22 \#3009)

[33] K. Kodaira and D. C. Spencer, On deformations of complex analytic structures. III. Stability theorems for complex structures, Ann. of Math. (2) 71 (1960), 43-76. MR0115189(22 \#5991)

[34] X. Masa, Duality and minimality in Riemannian foliations, Comment. Math. Helv. 67 (1992), no. 1, 17-27, DOI 10.1007/BF02566486. MR.1144611 (93g:53040)

[35] M. Min-Oo, E. A. Ruh, and P. Tondeur, Vanishing theorems for the basic cohomology of Riemannian foliations, J. Reine Angew. Math. 415 (1991), 167-174. MR1096904(92b:58218)

[36] P. Molino, Riemannian foliations, Progress in Mathematics, vol. 73, Birkhäuser Boston Inc., Boston, MA, 1988. Translated from the French by Grant Cairns; With appendices by Cairns, Y. Carrière, É. Ghys, E. Salem and V. Sergiescu. MR932463 (89b:53054)

[37] P. Molino and V. Sergiescu, Deux remarques sur les flots riemanniens, Manuscripta Math. 51 (1985), no. 1-3, 145-161, DOI 10.1007/BF01168350 (French, with English summary). MR788676 (86h:53035)

[38] H. Nozawa, Continuity of the Álvarez class under deformations, J. Reine Angew. Math. 673 (2012), 125-159.

[39] L. Ornea and M. Verbitsky, Structure theorem for compact Vaisman manifolds, Math. Res. Lett. 10 (2003), no. 5-6, 799-805. MR2024735(2004j:53093)

[40] L. Ornea and M. Verbitsky, Locally conformal Kähler manifolds with potential, Math. Ann. 348 (2010), no. 1, 25-33, DOI 10.1007/s00208-009-0463-0. MR2657432 (2011m:53138)

[41] J. I. Royo Prieto, The Euler class for Riemannian flows, C. R. Acad. Sci. Paris Sér. I Math. 332 (2001), no. 1, 45-50, DOI 10.1016/S0764-4442(00)01770-5 (English, with English and French summaries). MR 1805626 (2001m:53048)

[42] H. Rummler, Quelques notions simples en géométrie riemannienne et leurs applications aux feuilletages compacts, Comment. Math. Helv. 54 (1979), no. 2, 224-239, DOI 10.1007/BF02566270 (French). MR535057 (80m:57021)

[43] M. Saralegui, The Euler class for flows of isometries, Differential geometry (Santiago de Compostela, 1984), Res. Notes in Math., vol. 131, Pitman, Boston, MA, 1985, pp. 220-227. MR864872 (88a:58163)

[44] D. Sullivan, A homological characterization of foliations consisting of minimal surfaces, Comment. Math. Helv. 54 (1979), no. 2, 218-223, DOI 10.1007/BF02566269. MR535056 (80m:57022)

[45] T. Yamazaki, A construction of K-contact manifolds by a fiber join, Tohoku Math. J. (2) 51 (1999), no. 4, 433-446, DOI 10.2748/tmj/1178224713. MR1725620(2001e:53094)

Institut des Hautes Études Scientifiques, Le Bois-Marie 35, Route de Chartres 91440

Bures-Sur-Yvette, France

E-mail address: nozawahiraku@06.alumni.u-tokyo.ac.jp

Current address: Department of Mathematical Sciences, Faculty of Science and Engineering,

Ritsumeikan University, Nojihigashi 1-1-1, Kusatsu, Shiga, 526-8755, Japan 